\title{
10
}

\section{Bird, mammal and reptile remains}

\author{
Trevor H. Worthy \\ School of Biological, Earth and Environmental Sciences, The University of New South Wales \\ Geoffrey Clark \\ Department of Archaeology and Natural History, The Australian National University
}

\section{Introduction}

This chapter reports the non-fish remains from 10 archaeological excavations on Viti Levu and the Lau Group, including the reanalysis of a bird-bone assemblage from Lakeba Island excavated previously by Simon Best (1984). Bone remains from Natunuku and Ugaga were uncommon and the small assemblages were misplaced during collection relocation after bushfires destroyed the ANU archaeological storage facility in 2003, and these assemblages are not considered further. Three of the non-fish faunal assemblages are from the Lau Group (Qaranipuqa, Votua, Sovanibeka), one is from the north coast of Viti Levu (Navatu 17A), and the remainder are from the southwest Viti Levu region (Malaqereqere, Tuvu, Volivoli II, Volivoli III, Qaraninoso II) and Beqa Island (Kulu). This chapter presents the non-fish fauna from the Lau Group, followed by that from Viti Levu and Beqa Island.

Faunal analysis began early in Fiji, with bone remains identified at Navatu and Vuda on Viti Levu by Gifford (1951:208-213). Gifford's excavations demonstrated that pig, dog, chicken, turtle, fruit bat and humans were consumed during the 'early period' of Fiji. The study of archaeofauna declined after this promising start due to the absence of prehistoric fauna in sites such as Sigatoka and Karobo (Palmer 1965; Birks 1973), and the cursory identification of bone remains at sites like Yanuca and Natunuku (Birks and Birks 1978; Davidson et al. 1990), which was, in part, a result of the intense focus on the decorative system found on Lapita ceramics. Although a detailed analysis of prehistoric fauna from Lakeba was made by Best (1984), it is notable that Gifford's (1951) faunal identifications were the only detailed archaeofaunal record available for the large island of Viti Levu before the EPF investigations in the 1990s. As Viti 
Levu and Vanua Levu are significantly larger and older landforms than the islands of the Lau Group, it was thought probable that west Fiji contained different taxa than east Fiji, and human arrival and transformation of the environment may have followed a different path on the two large islands than on the small islands of the Lau Group.

Consequently, it was anticipated that the non-fish fauna from prehistoric sites in Fiji would shed light on the nature and variability of Lapita subsistence systems - in particular, the effect Lapita people had on Fiji's native birds and reptiles through predation and the introduction of commensal species such as the pig, dog, chicken and rat. The status of these species as Lapita imports has been unclear for many years, with a pig bone from basal levels of Yanuca (Hunt 1980) now identified as turtle, and a dog bone from Naigani (Best 1981) now regarded as questionable (Worthy pers. obs.). In Tonga, only the domestic fowl (Gallus gallus) and commensal Pacific rat (Rattus exulans) are unequivocally identified as Lapita-era introductions, raising the possibility that Lapita economies in Fiji-West Polynesia were initially less reliant on transported plants and animals for subsistence than those in the west (as outlined in Clark and Anderson 2001; Burley 2007). The second issue concerned the nature of post-Lapita subsistence and whether it involved a broadening of diet items, perhaps as a response to the loss of large and readily available wild-food resources, coupled with the increasing food demands of a growing population. Such conditions could result in food stress and relate to social adaptations in the Fiji sequence, including settlement pattern change and increased investment in horticultural production systems, as well as greater inter-group conflict and cannibalism.

\section{Methods}

Bird, reptile and mammal remains were identified by THW and GC using archaeozoology reference collections held at the ANU and the Museum of New Zealand. Worthy borrowed from the Fiji Museum the collection of bird bones excavated from the Qaranipuqa rock shelter (Best 1984). He identified them in the Museum of New Zealand Te Papa Tongarewa, with reference to the extensive collection of modern material housed there, augmented by study of loaned skeletal material from the Fiji Museum, the Smithsonian Institution (USNM), and the Canterbury Museum (CM). NISP and MNI were calculated for all assemblages, but only NISP is reported due to the degree of deposit mixing and the small amount of non-fish fauna in most sites. The exception is the bird remains from a stratified site on Lakeba for which an MNI and a maximum MNI were calculated for each level of the Qaranipuqa rock shelter. A recent study has found unexpected diversity amid Fijian iguanas and has named a third species Brachylophus bulabula for populations in the wet forests of Viti Levu, Vanua Levu and Kadavu, formerly known as B. fasciatus (Keogh et al. 2008). Populations in the Lau Group remain as B. fasciatus. We follow this nomenclature here.

\section{The Lau Group}

\section{Reanalysis of Lakeba avifauna: The Best collection}

In the late 1970s, Simon Best directed excavations at two adjacent sites on the northwest coast of Lakeba, and recovered a still unparalleled collection of Fijian archaeofauna, particularly bird remains, spanning Lapita and subsequent cultural phases (Steadman 2006a). The sites were Qaranipuqa (101/7/197), a large rock shelter eroded out of the limestone cliffs $220 \mathrm{~m}$ from the sea, and Wakea (101/7/196), a raised sand plain at the base of the limestone cliffs, just east of Qaranipuqa. The excavations recovered 1225 bird bones and fragments, most of which came from the three lowest layers of the Qaranipuqa rock shelter in association with Lapita pottery. 
In total, 21 species from 11 families were identified by P. Millener, G. van Tets, S. Olsen and D. Steadman. Identifications were reported in Best's PhD thesis (Best 1984:Table 7.7), and included an extinct megapode attributed to Megapodius freycinet and an extinct large pigeon (Ducula sp.).

The great advantage of the Lakeba collection of late-Holocene avifauna is that it derives from one of the few well-stratified, carefully excavated and adequately radiocarbon-dated Lapita sites in Fiji. However, the initial identifications given in Best's 1984 thesis now require revision. Best $(1984: 528-532,2002)$ noted that the comparative material available to identify pigeons, doves and rails was restricted and in some cases attribution to species/family was 'fairly general'. The lack of detailed comparative material with which to compare the Lakeba bird bones resulted in the tentative identification of two pigeons, two parrots and the barred-wing rail, and raised the possibility that two specimens of Gallus gallus in Lapita levels might belong to Megapodius freycinet. The last point is significant, as an incorrect Gallus identification might favour the view of a colonising population without domestic animals, in line with Groube's (1971:312) much contested view of Lapita people as 'oceanic strandloopers' who had a restricted maritime/ lagoonal economy (see Davidson and Leach 2001).

Recent work has obtained an enlarged fossil record for Fiji (Worthy 2000, 2001a, 2001b; Worthy and Anderson 1999; Worthy et al. 1999; see Chapters 2-3). These palaeofaunal investigations and a larger set of modern reference specimens have allowed a more accurate species list for the Lakeba avifauna. The revised list includes records of several species previously unknown in Fiji and returns a significant increase in the number of species (see below). The principal archaeological benefit of the revision lies in its contribution to understanding the nature of the Lapita economy in east Fiji. For several reasons, such as a high degree of deposit disturbance and the relatively coarse excavation techniques used in the past, this aspect of the Fijian sequence is not well understood on Viti Levu (see Clark and Anderson 2001). Thus the Lakeba cultural sequences excavated by Best are currently the best example of the marked impact of prehistoric people on Fiji's land-bird populations during the colonisation phase.

\section{Skeletal elements and descriptive terms}

The following abbreviations apply to single and plural usage of the elements: $\mathrm{cmc}$, carpometacarpi; cor, coracoids; fem, femora; fib, fibulae; hum, humeri; pt, part; quad, quadrates; rad, radii; scap, scapulae; stern, sterna; tmt, tarsometatarsi; and tib, tibiotarsi. When listing material, bones are sometimes identified as left (L) or right (R) elements. L or R prefixed by 'p', 's', or 'd' indicates 'proximal', 'shaft', or 'distal' part of the element respectively, e.g. $\mathrm{pR}$ fem means the proximal part of a right femur.

Anatomical nomenclature for specific bone landmarks follows Baumel and Witmer (1993), but terms are Anglicised after first mention. Some common terms are abbreviated as follows: proc. for processus; artic. for articularis.

\section{Systematics}

The Best collection from Lakeba contained many fragmentary bones, however 304 bones were identified to a taxon as recorded below (each element and catalogue number separated by semicolon), with comments on their identification if necessary. In the catalogue number (recorded on the bones), the first three digits refer to the site (197). The excavation at this site had two squares called Square 1 and Square 3, identified in the following digit after 197. The alphanumeric (e.g. $\mathrm{W}$ or $\mathrm{T}$ ) refers to the layer (top is $\mathrm{A} 1$, bottom is $\mathrm{X}$, in total 34 layers). Layer T1 and T2 are about $4 \mathrm{~m}$ below surface, and Layers W1 and W2 are approximately $4.5 \mathrm{~m}$ below the surface and are 
the earliest cultural layers of the site. Best (1984) divided the layers into parts, such as T1 or T2, and so the digit after the letter in the catalogue number is assumed to be the layer part, which can be as high as 7 for layer $M$ or 10 for layer K. Here, the specimens are tabulated into layers A to $U$ only (Table 37), and even then, single bones had constituent parts in adjacent layers, so finer tabulation is not warranted for faunal analysis.

While most bones (300) are from Qaranipuqa, a few are from other sites, and for completeness they are listed with the rest of the Lakeba fauna. Two bones are from Site 196, or Wakea, which is adjacent to the Qaranipuqa site. They are the single bone of Egretta sacra and one of the two Limosa lapponica bones, so are significant additions to the total fauna. One bone of Columba vitiensis is from Site 47, which is the fortified site Ulunikoro or Vagadra above Qaranipuqa. One bone of Porphryio porphyrio (135.1.3.C+D) is from a ring-ditch settlement known as Liqau on the northwest coast. All are listed below as a complete listing of identified Lakeba material from Best's excavations (Table 37).

\section{Class Reptilia}

\section{Family Boidae}

Candoia bibroni Pacific boa

Material: vertebra, 197.1.X.2,7.

\section{Class Aves}

\section{Family Phaethontidae}

Phaethon lepturus White-tailed tropicbird

Material: R cmc, 197.3.A.5(a), 1; R cor, 197.3.N.1(e), 3; L scap, 197.3.N.1(e), 7; L tib, 197.1.A.3.4, 1; pL cor, 197.1.N.1.3, 4; R rad, 197.1.M.10.4, f96, 1; L cmc, 197.1.M.8.3, f80, 1; d+sL rad, 197.3.M.10(a), 9; dL ulna, 197.3.M.8(e), 1; (total 9 bones).

\section{Family Fregatidae}

Fregata ariel Lesser frigatebird

Material: pR cor, 197.3.K.10(a), 1; (total 1 bone).

\section{Family Ardeidae}

Egretta sacra Reef heron

Material: pR hum, 196.18.A.3, 1; (total 1 bone).

Butorides striatus Mangrove heron

Material: dL tib, 197.3.W.1.7, 10; (total 1 bone)

\section{Family Anatidae}

Anas superciliosa Black duck

Material: sternal end L cor, 197.3.V.7, 1; dR ulna, 197.1.L.f18, 4; (total 2 bones).

\section{Family Megapodiidae}

Megapodius alimentum Extinct scrubfowl

Material: A total of 49 bones. The elements and their catalogue numbers are listed in Worthy (2000), except the sL cmc (197.1.U.2,4) that was subsequently identified. Nearly all were from layer W, with one from Layer $\mathrm{U}$, two from Layer $\mathrm{V}$ and three from Layer X. 
This species, first described on bones from Tonga by Steadman (1989), was redescribed by Worthy (2000) using the Lakeba material.

\section{Family Phasianidae}

Gallus gallus Domestic fowl

Material: dR hum, 197.3.M.1, 1; dL tib, 197.3.W.1.4, 12; dL tib, 197.3.U.1(E), 4; dL tib, 197.3.W.2E, 10; (total 4 bones).

\section{Family Rallidae}

Gallirallus philippensis Banded rail

Material: sL tib, 197.1.A.14.4, 1; R cor, 197.1.E.4,f1,f19, 32, 1 (medial length = $22.9 \mathrm{~mm}$ ); sternum, 197.1.M.4.3, 2; L cor, 197.2.M.1, 2 (medial length = 24.4 mm); pR tib, 197.3.H.4(e), 3; dR tib, 197.3.F.1.f171(d).a, 4; d+sR tmt, 197.3.F.f171(d).a, 2; R cor, 197.1.L.1.3, 2 (medial length $=22.4 \mathrm{~mm}$; R cor, 197.1.A.11.4, 1 (medial length = 21.5 mm); (total 9 bones).

These bones are listed by Steadman (2006a) as a flightless taxon 'Gallirallus undescribed sp. D', which was assumed to be endemic to Lakeba and nearby Aiwa Levu and Aiwa Lailai. However, the material listed here provides no evidence for the bones having been derived from a flightless taxon, or that they differed significantly from the widespread Gallirallus philippensis. Medial lengths for coracoids of two specimens of Gallirallus philippensis sethsmithi from Fiji are: FM8 $22.2 \mathrm{~mm}$; FM19 $23.7 \mathrm{~mm}$, well within the range of the fossils.

Porphyrio porphyrio Purple swamphen

Material: dL fem, 197.3.K.5(1), f226, 4; sR tib, 197.3.F.1, f171(6), 2; pt pR ulna, 197.3.M.7(2), 1; s+dR tmt, 135.1.3.C+D, leve SW; R fem, 197.1.L.4(a).1, 1; dR tib, 197.3.W.1.1, 29; pR hum, 197.3.K2.1.L4 NE extn, 1; sR tib, 197.3.M.7(a), 2; dR tmt, 197.1.x.1, 31; dR tmt, 197.3.K.10(L1.4), 2; dL tib, 197.1.L.3a.4; s+pL tib, 197.3.E.2+3(a), 1; R cor, 197.3.M.6(a), 1; dR tib 197.1.L.3a.4,3; pR cor, 197.3.W.1.2, 21; sR tib, 197.1.M.1.3, 1; dR tib, 197.1.J.2.1, 1; pR fem, 197.1.N.1.3, 3; pR cor, 197.1.C.3a.4, 2; dL tib, 197.3.K.10(L1-4), 4; sR hum, 197.3.M.10, f238, 1; dL cmc, 197.1.N.1.3(b), 1; dR tib, 197.1.L.3(c).1, 2; pt pR hum, 197.1.L.3a.4, 1; L cor, 197.3.W.1.1, 9; R cor, 197.3.K.2(a), 2; dL ulna, 197.3.W.1.1, 18; dL rad, 197.3.W.1.1, 27; dR tib, 197.3.W.2(E), 31; L scap, 197.3.W.1.4, 17; sR tmt, 197.3.K.10(L1-4), 5; dR tmt, 197.3.W.3(w), 2; sR tmt, 197.3.K.10(L1-4), 4; dR tmt, 197.1.M.9.1, 8; dL fem, 197.1.M.9.1, 10; sternal end L cor, 197.3.W.2, 26; pR ulna, 197.3.O.1(E), 1; d+sR tib, 197.3.E.5, 4; sL tib, 197.3.H.2(f), 1; sR fem, 197.3.F.1(a), 2; L cor, 197.1.M.9.2, 1; (total 41 bones).

These bones are of the small Pacific form of Porphyrio, often listed in the subspecies $P . p$. vitiensis or $P$. p. samoensis. They are distinct from the Australasian $P$. melanotus. The smaller examples of this species from this site were listed previously by Best (1984) as Nesoclopeus poicilopterus.

Porzana tabuensis Spotless crake

Material: L hum, 197.3.E.2+3, 1; (total 1 bone).

\section{Family Charadriidae}

Pluvialis fulva Pacific golden plover

Material: L cor, 197.1.E.4.3, 1; (total 1 bone). 


\section{Family Scolopacidae}

Limosa lapponica Bar-tailed godwit

Material: pt sternum, 196.17.B.4, f14; dR hum, 197.3.A.5, 3; (total 2 bones).

\section{Family Columbidae}

Gallicolumba stairii Friendly ground dove

Material: L scap, 197.3.W.1.8, 45; L scap, 197.3.W.1.8, 12; R cor, 197.1.T.6, 2; dR ulna, 197.3.?; sR tib, 197.1.W.1(e), 6; dL tmt, 197.3.T.6, 1;pL hum, 197.3.W.1.6, 2; dR cmc, 197.1.T.6, 3; (total 8 bones).

These bones conform well with a large series from Vatulele.

Didunculus strigirostris Tooth-billed pigeon

Material: L quad 197.1.W.2, 30; pR cmc, 197.3.W.1.8; pL tmt, 197.3.W.1.8, 39; (total 3 bones).

These bones are good matches for CM Av7160. The carpometacarpus has a more upturned and pointed proc. extensorius than in Ducula, and it is grooved proximally between the minor and major os metacarpales on the dorsal surface. The tarsometatarsus is more elongate than Ducula or Columba and has a characteristic very small foramina vascularia proximalia lateralis.

?Didunculus sp.

Material: pR cor, 197.3.W.1.2, 10; dL cor, 197.3.w?, 11; L hum, 197.1.v.1(c), 13; dR cor, 197.3.W.3, 2; pR cor, 197.3.W.7.3, 29; (total 5 bones).

The proximal coracoids are tentatively referred to Didunculus because they are highly pneumatic under the acrocoracoid, and the distal coracoid has a large dorsal pneumatic fossa, but they are not Caloenas. These features exclude Ducula and Columba and the bones are of the expected size for Didunculus. CM Av7160 lacks comparable elements, precluding certain identification of these fossils.

Ducula lakeba Lakeba pigeon

Material: total 92 bones. The elements and their catalogue numbers are listed in Worthy (2001b). Most specimens are from Layer W. Two are from Layer X, 13 from Layer V, and one from Layer T, i.e. in the basal Lapita layers of the site. This species was described by Worthy (2001b) and named for the island from which it was first identified. However, it is a volant pigeon and is expected to have occurred on all the larger islands of the Lau Group. A similar, if not the same species, occurred on Viti Levu as well. It is slightly larger and more gracile than Ducula david described from Wallis Island by Balouet and Olson (1987).

Ducula pacifica Pacific pigeon

Material: d+sR hum, 197.3.W.1(a), 2; pR cmc, 197.1.L.3(c).1, 1; pL cmc, 197.1.W.2, 38; L tmt, 197.1.W.1, 24 + 197.1.V.2, 1; pR cor, 197.3.W.1(a), 5; pR cor, 197.3.U.2, 7; (total 6 bones).

The tarsometatarsus of $D$. pacifica is longer than Columba vitiensis, and has a large lateral vascular foramen (much reduced in Columba). On the coracoid, the acrocoracoid is not dorsally expanded to markedly overhang the sulcus supracoracoidei as in Columba. Carpometacarpus with proc. alularis more distad than internal rim of trochlea carpalis (equal in Columba). 
Ducula pacifica or Columba sp.

Material: dR cmc, 197.3.W.1.8, 22; R scap, 197.3.W.1(a), 6; sR ulna, 197.3.V.1(E), 2; (total 3 bones). Bones were not sufficiently complete for further identification.

Columba vitiensis White-throated pigeon

Material: dR tib, 197.1.W.1, 11; pelvis, 197.3.K.6+7(a), 1; pR cmc, 197.3.W.1.B; dL tmt, 197.1.W.2, 1; dR tib, 197.1.W.1, 31; sR tib, 197.3.V.1(E), 1; pL cor, 197.1.W.1, 16; pR scap, 47.10.B(5-), 1; (total 8 bones).

Bones of Columba vitiensis are smaller than Ducula pacifica and otherwise differ by: tarsometatarsi with straight lateral margin of shaft (not concave), and a more pointed trochlea metatarsi II (medial trochlea). Carpometacarpus with alular process and internal rim of carpal trochlea of equal distal extent (not with alular process more distad as in Ducula). Tibiotarsus with lateral condyle more rounded cranio-proximally. Coracoid with acrocoracoid markedly overhanging the supracoracoidal sulcus (does not in Ducula). Scapula with pneumatic acromion (not in Ducula).

Columbid sp. Unidentified pigeon

Material: L cmc, 197.3.W.1.8, 1 (length $=39.44 \mathrm{~mm}$, proximal width $=10.6 \mathrm{~mm}$ ); pR cmc, 197.3.W.1.1, 2; dL cmc, 197.1.W.1, 10; pt sL tib 197.3.K2 to L4 NE ext, 7; L rad 197.3.W.1.6; pR rad, 197.3.W.1.6, 117; dL cor 197.3.W.2, 27; pt head of R hum, 197.3.W.1.5, 41; L scap, 197.3.W.2(E), 9; sL hum, 197.1.w, 1; dL cor 197.3.W.1.8, 2; dR hum 197.3.W.1.6, 26; sR hum 197.3.A.15(a), 1; dL fem 197.1.W.B1, 11 (distal width = 9.16 mm); sL fem 197.1.W.2, 41; pL scap 197.1.W.1, 35; L scap 197.3.v.3(w), 2; (total 17 bones).

Most of these specimens are bigger than the available Ducula pacifica reference skeletons, or the same size or bigger than the larger D. latrans skeleton at hand (FM28). It is probable they represent larger specimens of one or both these species. There is insufficient skeletal material of $D$. latrans to adequately define the size range of elements and no qualitative differences in postcranial elements were noted. Steadman (2006a) lists D. latrans from his excavations on Aiwa Levu and Aiwa Lailai.

Ptilinopus porphyraceus Crimson-crowned fruit dove

Material: dL ulna, 197.1.S.3.2, 1; L cmc, 197.3.W.1.1, 17; dR cor, 197.1.x.1, 6; dL fem, 197.3.W.1.1, 11; sL hum, W.3(w); R tmt, 197.3.V.3, 1; pR hum, 197.1.W.1, 4; dR tib, 197.3.W.1.4; dL tib, 197.3.W.1, 33; sL cor, 197.3.T.7, 8; R cmc, 197.3.W.1(a), 3; dR ulna, 197.3.W.1.1, 59; R ulna, 197.3.W.1(a), 8; (total 13 bones).

Ptilinopus sp. Undetermined dove species

Material: R tmt, 197.3.W.1.5, 23; (total 1 bone).

The specimen lacks the distal trochlea but with a length of $22.5 \mathrm{~mm}$ to the middle of the distal foramen and an estimated length of $26 \mathrm{~mm}$, it is much longer than $P$. porphyraceus or $P$. perousii, and any members of the golden dove (P. lutiovirens, $P$. victor, P. layardi) group of Fiji. However, measurements given by Steadman (1992) show that both P. rarotongensis of Rarotonga and $P$. purpuratus of the Society Islands-Tuamotu Group have similar sized tarsometatarsi. 


\section{Family Psittacidae}

Prosopeia sp. Undetermined species of musk parrot

Material: sL tmt, 197.1.X.1, 30; dL ulna, 197.3.T.6(E), 1; dL hum, 197.1.V.1(a), 1; (total 3 bones).

These bones were bigger than P. tabuensis splendens (USNM 614998), but this sub-species from Kadavu may be smaller than other subspecies ( $P$. $t$. atrogularis from Vanua Levu and offshore islands, $P$. t. taviunenesis from Taveuni and Qamea, $P$. t. koroensis from Koro, and $P$. $t$. tabuensis from Gau and Tonga), for which no skeletons were available.

Vini solitarius Collared lory

Material: pL ulna, 197.3.W.1.4, 4; dL hum, 197.3.T.7, 1; R tmt, 197.3.W.1.6, 33; R cmc, 197.3.W.1.6, 7; pR ulna, 197.3.W.1.4, 7; L cor, 197.3.W.1.6, 41; R rad, 197.3.T.6, 11; R scap, 197.3.W.2; (total 8 bones).

?Charmosyma amabilis ?Red-throated lorikeet

Material: L tib, 197.3.F.1; f189; 5 (length $=$ ca. $28.3 \mathrm{~mm}$, proximal width $=3.26 \mathrm{~mm}$, shaft width $=1.39 \mathrm{~mm}$.); (total 1 bone).

No reference specimen available.

\section{Family Tytonidae}

Tyto alba Barn owl

Material: pt L cor, 197.1.L.4(a).1, 3; pR fem, 197.1.A.8.2, 1; dL tib, 197.3.E.4, 2; dR fem, 197.1.E.3+4, 1; (total 4 bones).

\section{Family Alcedinidae}

Halcyon chloris White-collared kingfisher

Material: L cmc, 197.1.A.12.1; dR ulna, 197.1.A.4.2, 2; (total 2 bones).

\section{Family Meliphagidae}

Foulehaio carunculata Wattled honeyeater

Material: L tib, 197.3.K.2(a).1, 1; R cmc, 197.1.V.1(e), 14; L hum, 197.1.A.4.2, 1; (total 3 bones).

\section{Family Sturnidae}

Aplonis tabuensis Polynesian starling

Material: R ulna, 197.1.A.6.4, 2 ; R tmt, 197.1.E.9, 32, ( $\mathrm{L}=26.05 \mathrm{~mm})$; dR tib, 197.3.M.11, E; R rad, 197.1.A.6.4, 3; L hum, 197.1.A.6.4, 1; (total 5 bones). 
Table 37. The faunal list derived from 302 identified bones from the adjacent sites Qaranipuqa (site 197) and Wakea (site 196) on Lakeba. Only the single Egretta sacra bone and one Limosa bone are from Site 196. Data is shown as NISP (number of specimens), MNI (minimum number of individuals for whole assemblage), NISP within Layers A-U, and Max MNI (MNI maximised by assuming sets of layers come from single units, as follows $\mathrm{U}-\mathrm{X}$, and $\mathrm{H}-\mathrm{O}, \mathrm{E}-\mathrm{F}$, and $\mathrm{A}-\mathrm{C}$ ).

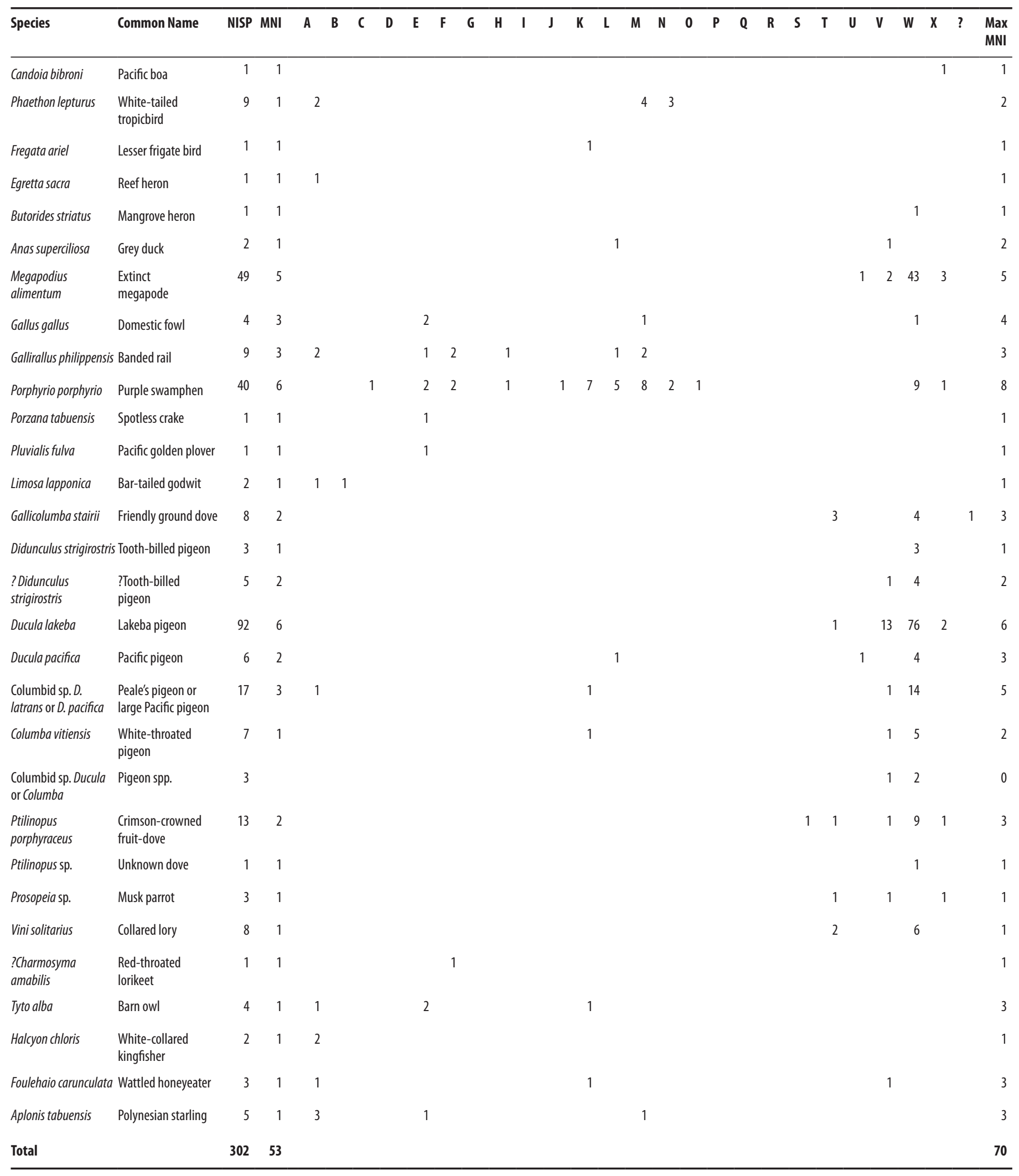




\section{Votua and Sovanibeka (Mago Island, Lau Group)}

The Votua Lapita site was re-excavated in 2000, and the water-sieved sediments from the Area 2 trench excavation ( $1 \mathrm{~m} \times 4 \mathrm{~m}$ ) yielded small amounts of identifiable bird, rat and turtle bone (Table 38). Land birds were hunted at Votua by Lapita people, as at Lakeba, with remains recovered from an extinct megapode, Megapodius alimentum, banded rail and Polynesian starling. The passerine, Gymnomyza viridis (giant forest honeyeater), has been recorded in modern times on Viti Levu, Vanua Levu and Taveuni, but is not found in the Lau Group today (Steadman 2006a). As it occurs at Votua, it may have been more widely distributed in the Fiji Islands in the past. A humerus from a young sea turtle (Chelonia mydas) and rat bones from Rattus exulans and Rattus praetor were also recovered. Two $R$. praetor bones were sent for AMS analysis to determine whether the spiny rat arrived in Fiji in Lapita times, or was introduced from the west at a later date (see White et al. 2000). However, after pre-treatment, both the rat bones failed to provide sufficient material for an age determination, and as the burrowing propensity of this rat is unclear (Taylor et al. 1982), the remains could post-date the Lapita occupation at Votua.

Fauna from the Sovanibeka Test Pit 2 excavation derived from an owl-roost deposit and prehistoric human activity (Table 38). The remains of $R$. exulans and $R$. praetor were abundant, along with very small bones from the swiftlet (Collocalia spodiopygia) and the Oceanic gecko (Gehyra oceanica), both residents of the limestone complex today. An AMS date on a wellpreserved $R$. praetor bone had an age of 670-770 cal. BP (OZF882), evidence that the spiny rat lived in Fiji during the second millennium $\mathrm{AD}$, before apparently becoming extinct. Land snails were common in the lower part of the Sovanibeka deposit, but diminished in number in the upper stratigraphy, with four species identified using the comparative collection of the Australian Museum (Gonatorhaphe lavensis, Helicina tectiformis, Truncatella cf. clathrata and Omphalotropsis sp.) A ${ }^{14} \mathrm{C}$ result on land-snail shell (Gonatorhaphe lavensis) from Test Pit 1 had an age of $4290 \pm 60 \mathrm{BP}$ (ANU-11246). Owl predation is almost certainly responsible for the sheathtailed bat (Emballonura semicaudata) and banded iguana (Brachylophus bulabula) remains, while burnt turtle bone and bone from the domestic fowl probably result from prehistoric human use of the shelter. Sparse dentate-stamped and paddle-impressed sherds and occasional food debris, along with use of the shelter as an owl roost, suggest intermittent use of the Sovanibeka site in prehistory.

\section{Viti Levu and Beqa Island}

\section{Navatu 17 A (north Viti Levu)}

The non-fish bone assemblage from the 1996 excavation of Trench B (Squares 1-4) had a NISP of 252 (Table 39). The bird bone was small and broken, consisting of shaft fragments from limb bones, and only two bones from the barn owl (Tyto alba) were able to be identified. Barn owls are primarily birds of open country or forest edges, and are specialist rodent predators. Prehistoric human colonisation of the Pacific was responsible for introducing rats (Rattus exulans and Rattus praetor) and vegetation clearance, both of which would have enabled owls to expand their range after human colonisation. The presence of owl at Navatu suggests relatively open country surrounding the volcanic plug by 800 BP (ANU-10384). Bird bone from the upper levels (0-70 cm depth) of the 1947 excavation was identified as black duck (Anas superciliosa), barred-wing rail (Gallirallus (=Nesoclopeus) poicilopterus) and swamp harrier (Circus approximans) (Gifford 1951:211). None of the turtle or lizard bone was identifiable to species and both were present in small amounts. 
Table 38. Votua and Sovanibeka non-fish remains.

\begin{tabular}{|c|c|c|c|c|}
\hline Provenience & Taxa & Common name & Element & NISP \\
\hline \multicolumn{5}{|l|}{ Votua: Area 2} \\
\hline Square 3: 0-10 cm & Gallirallus philippensis & Banded rail & distal femur (L) & 1 \\
\hline Square 2: $10-20 \mathrm{~cm}$ & G. philippensis & Banded rail & distal femur (R) & 1 \\
\hline Square 4: $10-20 \mathrm{~cm}$ & Gallus?species & Scrub fowl & proximal manus phalange (R) & 1 \\
\hline Square: $3: 10-20 \mathrm{~cm}$ & G. philippensis & Banded rail & shaft tarsometatarsus (R) & 1 \\
\hline Square $3: 30-40 \mathrm{~cm}$ & Megapodius alimentum & Extinct scrubfowl & proximal ulna (L) & 1 \\
\hline Square $1: 30-40 \mathrm{~cm}$ & Halcyon sancta & White-collared kingfisher & distal humerus (R) & 1 \\
\hline Square 2: $30-40 \mathrm{~cm}$ & Chelonia mydas & Green turtle & radius (L) & 1 \\
\hline Square $3: 30-40 \mathrm{~cm}$ & Rattus praetor & Spiny rat & femur (R) & 1 \\
\hline Square 2: $40-50 \mathrm{~cm}$ & Megapodius?species & Scrub fowl & proximal radius $(\mathrm{R})$ & 1 \\
\hline Square 4: $50-60 \mathrm{~cm}$ & cf. Gymnomyza viridis & ?Giant forest honeyeater & proximal carpometacarpus (R) & 1 \\
\hline Square 4: $50-60 \mathrm{~cm}$ & Pteropus cf tonganus & Large fruit bat & distal humerus & 1 \\
\hline Square $3: 50-60 \mathrm{~cm}$ & Rattus praetor & Spiny rat & tibia (L) & 1 \\
\hline Square 4: $50-60 \mathrm{~cm}$ & Megapodius?species & Banded rail & distal pelvis & 1 \\
\hline Square $3: 50-60 \mathrm{~cm}$ & G. philippensis & Banded rail & coracoid (L) & 1 \\
\hline Square 4: $50-60 \mathrm{~cm}$ & Ratus exulans & Pacific rat & mandible (L) & 1 \\
\hline Square 2: $50-60 \mathrm{~cm}$ & Megapodius alimentum & Extinct scrubfowl & distal ulna (R) & 1 \\
\hline Square 4: $50-60 \mathrm{~cm}$ & cf. Aplonis tabuensis & Polynesian starling & proximal ulna (L) & 1 \\
\hline Square 4: $60-70 \mathrm{~cm}$ & Megapodius ?species & Scrub fowl & shaft femur (L) & 1 \\
\hline \multicolumn{4}{|l|}{ NISP Total } & 18 \\
\hline \multicolumn{5}{|l|}{ Sovanibeka Test Pit 2} \\
\hline TP 2: 0-50 cm & Collocalia spodiopygia & Swiftlet & all elements present & $>60$ \\
\hline TP 2: 0-50 cm & Rattus exulans & Pacific rat & all elements present & $>60$ \\
\hline TP 2: 0-50 cm & Rattus praetor & Spiny rat & all elements present & $>40$ \\
\hline TP 2: 0-30 cm & Porphyrio porphyrio & Purple swamphen & proximal carpometacarpus (R) & 1 \\
\hline TP 2: $0-30 \mathrm{~cm}$ & Columbid species & Pigeon sp. & sacrum & 1 \\
\hline TP 2: 0-30 cm & Halcyon chloris & White-collared kingfisher & humerus (R) & 1 \\
\hline TP 2: 0-30 cm & Aplonis tabuensis & Polynesian starling & $\begin{array}{l}\text { humerus }(\mathrm{L}+\mathrm{R}) \text {, tarsometatarsus }(\mathrm{R}) \text {, ulna }(\mathrm{R}) \text {, } \\
\text { tibiotarsus }(\mathrm{L})\end{array}$ & 5 \\
\hline TP 2: 0-30 cm & ?Gallicolumba & Dove species & proximal tibia (R) & 5 \\
\hline TP 2: 0-30 cm & Porzana tabuensis & Spotless crake & $\begin{array}{l}\text { proximal humerus }(\mathrm{L}) \text {, tarsometatarsus }(\mathrm{R}) \text {, femur }(\mathrm{R}) \text {, } \\
\text { distal tibia }(\mathrm{R}+\mathrm{L})\end{array}$ & 5 \\
\hline TP 2: 0-30 cm & Gehyra oceanica & Oceanic gecko & maxilla $(2 \mathrm{R}+2 \mathrm{~L})$, dentary $(7 \mathrm{~L}+1 \mathrm{R})$ & 12 \\
\hline TP 2: 0-30 cm & Chelonia mydas & Green turtle & humerus & 1 \\
\hline TP 2: 0-30 cm & Gecko?species & Gecko species & $\begin{array}{l}\text { dentary ( } 3 L+1 R) \text {, scapulocoracoid (1R), femur (4), } \\
\text { humerus (1), maxilla ( } L+R)\end{array}$ & 12 \\
\hline TP 2: 0-30 cm & ?Emballonura semicaudata & Pacific sheath-tailed bat & dentary & 1 \\
\hline TP 2: $30-50 \mathrm{~cm}$ & Brachylophus fasciatus & Banded iguana & maxilla (L) & 1 \\
\hline TP 2: $30-50 \mathrm{~cm}$ & Halcyon chloris & White-collared kingfisher & humerus (L) & 1 \\
\hline TP 2: $30-50 \mathrm{~cm}$ & Gallus gallus & Domestic fowl & humerus $(R)$, shaft ulna & 2 \\
\hline TP 2: $30-50 \mathrm{~cm}$ & Porphyrio porphyrio & Purple swamphen & proximal humerus (R), distal femur (R), scapula (L) & 3 \\
\hline TP 2: $30-50 \mathrm{~cm}$ & Gallus gallus & Domestic fowl & distal tarsometatarsus (R), coracoid (R) & 2 \\
\hline TP 2: 30-50 cm & Aplonis tabuensis & Polynesian starling & $\begin{array}{l}\text { shaft humerus }(\mathrm{L}) \text {, distal ulna }(\mathrm{R}+\mathrm{L}) \text {, tibiotarsus }(\mathrm{R}) \text {, } \\
\text { tarsometatarsus }(\mathrm{L})\end{array}$ & 5 \\
\hline
\end{tabular}


242 Trevor H. Worthy and Geoffrey Clark

Table 39. Navatu 17A, Trench B, non-fish remains.

\begin{tabular}{|c|c|c|c|c|}
\hline Provenience & Taxa & Common name & Element & NISP \\
\hline $1: 0-20 \mathrm{~cm}$ & Homo sapiens & human & crania-vault & 2 \\
\hline $1: 20-40 \mathrm{~cm}$ & Mammal ?species & & fragment ( 2 burnt) & 3 \\
\hline $1: 20-40 \mathrm{~cm}$ & Mammal ?species & & fragment & 4 \\
\hline $2: 20-40 \mathrm{~cm}$ & Mammal ?species & & fragment & 1 \\
\hline $3: 20-40 \mathrm{~cm}$ & Sus scrofa & pig & calcaneum (R) & 1 \\
\hline $3: 20-40 \mathrm{~cm}$ & Homo sapiens & human & metatarsal-5 (R) & 1 \\
\hline $1: 40-50 \mathrm{~cm}$ & ?Mammal & & long bone & 1 \\
\hline $2: 40-50 \mathrm{~cm}$ & Homo sapiens & human & capitate & 1 \\
\hline $1: 40-50 \mathrm{~cm}$ & Mammal ?species & & metatarsal & 1 \\
\hline 1: $50-60 \mathrm{~cm}$ & ?Homo sapiens & & fragment & 1 \\
\hline $2: 50-60 \mathrm{~cm}$ & Turtle & & plastron & 4 \\
\hline $2: 50-60 \mathrm{~cm}$ & Homosapiens & human & Homo sapiens & 1 \\
\hline $2: 50-60 \mathrm{~cm}$ & Mammal ?species & & fragment & 1 \\
\hline $3: 50-60 \mathrm{~cm}$ & Mammal & & long bone fragment & 1 \\
\hline $1: 60-70 \mathrm{~cm}$ & Mammal ?species. & & fragment & 2 \\
\hline $3: 60-70 \mathrm{~cm}$ & Bird ?species & & long bone fragment & 1 \\
\hline $3: 60-70 \mathrm{~cm}$ & Homo sapiens & human & crania-parietal & 3 \\
\hline $3: 60-70 \mathrm{~cm}$ & Pteropus sp. & fruit bat & mandible & 1 \\
\hline $1: 70-80 \mathrm{~cm}$ & Mammal ?species & & proximal rib & 1 \\
\hline $1: 70-80 \mathrm{~cm}$ & Mammal ?species & & long bone & 2 \\
\hline $2: 70-80 \mathrm{~cm}$ & Turtle & & plastron & 1 \\
\hline $2: 70-80 \mathrm{~cm}$ & Homo sapiens & human & proximal radius & 1 \\
\hline $2: 70-80 \mathrm{~cm}$ & Homo sapiens & human & tooth-M' & 1 \\
\hline $2: 70-80 \mathrm{~cm}$ & Reptile ?sp. & & epiphysis & 1 \\
\hline $2: 70-80 \mathrm{~cm}$ & Bird?species & & long bone fragment & 1 \\
\hline $3: 70-80 \mathrm{~cm}$ & Mammal ?species & & shaft rib & 2 \\
\hline $4: 70-80 \mathrm{~cm}$ & Homo sapiens & human & proximal ulna & 1 \\
\hline $2: 80-90 \mathrm{~cm}$ & Lizard?Sp & & long bone fragment & 1 \\
\hline $2: 80-90 \mathrm{~cm}$ & Turtle? & & metatarsal & 1 \\
\hline $2: 80-90 \mathrm{~cm}$ & Turtle & & plastron & 1 \\
\hline $2: 80-90 \mathrm{~cm}$ & Mammal ?species & & fragment & 1 \\
\hline $3: 80-90 \mathrm{~cm}$ & Bird?species & & long bone fragment & 1 \\
\hline $3: 80-90 \mathrm{~cm}$ & ?Homo sapiens & human & shaft rib & 1 \\
\hline $3: 80-90 \mathrm{~cm}$ & Mammal ?species & & fragment & 1 \\
\hline 1: $90-100 \mathrm{~cm}$ & Homo sapiens & human & shaft ulna/radius & 1 \\
\hline 2: $90-100 \mathrm{~cm}$ & Turtle & & plastron & 2 \\
\hline 2: $90-100 \mathrm{~cm}$ & Bird?species & & long bone fragment & 1 \\
\hline 3: 90-100 cm & Turtle & & plastron & 1 \\
\hline 3: 90-100 cm & Rattus exulans & Pacific rat & humerus (L) & 1 \\
\hline $3: 90-100 \mathrm{~cm}$ & Homo sapiens & human & phalange-5 & 1 \\
\hline 3: 90-100 cm & Mammal ?species & & fragment & 1 \\
\hline
\end{tabular}


Table 39 continued

\begin{tabular}{|c|c|c|c|c|}
\hline Provenience & Taxa & Common name & Element & NISP \\
\hline $3: 100-110 \mathrm{~cm}$ & Pteropus sp. & fruit bat & mandible (R) & 1 \\
\hline $3: 100-110 \mathrm{~cm}$ & Mammal ?species & & fragment & 5 \\
\hline $1: 100-110 \mathrm{~cm}$ & Tyto alba & barn owl & distal tarsometatarsus (L) & 1 \\
\hline $2: 100-110 \mathrm{~cm}$ & Bird?species & & long bone fragment & 2 \\
\hline $3: 110-120 \mathrm{~cm}$ & Tyto alba & barn owl & distal ulna (R) & 1 \\
\hline $3: 110-120 \mathrm{~cm}$ & Bird?species & & long bone fragment & 1 \\
\hline $3: 110-120 \mathrm{~cm}$ & Bird?species & & distal tarsometatarsus & 1 \\
\hline $1: 120-160 \mathrm{~cm}$ & Homo sapiens & human & tooth-M $\mathrm{M}_{1}$ (caries) & 1 \\
\hline $1: 120-160 \mathrm{~cm}$ & Mammal ?species & & long bone & 1 \\
\hline 1: $180-190 \mathrm{~cm}$ & Mammal ?species & & fragment & 2 \\
\hline 1: $180-190 \mathrm{~cm}$ & ?Turtle & & metatarsal/carpal & 1 \\
\hline $1+2: 200-210 \mathrm{~cm}$ & Homosapiens & human & crania-vault & 1 \\
\hline $1+2: 210-220 \mathrm{~cm}$ & Mammal ?species & & fragment & 1 \\
\hline $1+2: 210-220 \mathrm{~cm}$ & Bird ?species & & long bone fragment & 1 \\
\hline $1+2: 210-220 \mathrm{~cm}$ & Turtle & & plastron & 2 \\
\hline $1+2: 210-220 \mathrm{~cm}$ & Mammal ?species & & fragment & 4 \\
\hline $1+2: 220-230 \mathrm{~cm}$ & Turtle & & plastron & 4 \\
\hline $1+2: 220-230 \mathrm{~cm}$ & Mammal ?species & & plastron & 10 \\
\hline $1+2: 230-240 \mathrm{~cm}$ & Turtle & & plastron & 4 \\
\hline $1+2: 230-240 \mathrm{~cm}$ & Mammal ?species & & long bone fragment & 7 \\
\hline $1+2: 230-240 \mathrm{~cm}$ & Mammal ?species & & shaft rib & 2 \\
\hline $1+2: 230-240 \mathrm{~cm}$ & Mammal ?species & & vertebra & 10 \\
\hline $1+2: 230-240 \mathrm{~cm}$ & Mammal ?species & & fragment & 4 \\
\hline $3+4: 230-240 \mathrm{~cm}$ & Bird?species & & long bone fragment & 4 \\
\hline $3+4: 230-240 \mathrm{~cm}$ & Bird?species & & fragment & 1 \\
\hline $3+4: 230-240 \mathrm{~cm}$ & Homo sapiens & human & shaft tibia & 3 \\
\hline $3+4: 230-240 \mathrm{~cm}$ & Mammal ?species & & fragment & 60 \\
\hline $1+2: 240-250 \mathrm{~cm}$ & Homo sapiens & human & zygomatic & 1 \\
\hline $1+2: 240-250 \mathrm{~cm}$ & Homo sapiens & human & crania-vault & 1 \\
\hline $1+2: 240-250 \mathrm{~cm}$ & Turtle & & plastron & 3 \\
\hline $1+2: 240-250 \mathrm{~cm}$ & Bird?species & & long bone fragment & 1 \\
\hline $1+2: 240-250 \mathrm{~cm}$ & Mammal ?species & & fragment & 41 \\
\hline $3+4: 240-250 \mathrm{~cm}$ & Mammal ?species & & shaft rib & 1 \\
\hline $3+4: 240-250 \mathrm{~cm}$ & Mammal ?species & & fragment & 5 \\
\hline $1+2: 250-260 \mathrm{~cm}$ & Rattus exulans & Pacific rat & distal radius & 1 \\
\hline $1+2: 250-260 \mathrm{~cm}$ & Rattus exulans & Pacific rat & vertebra & 1 \\
\hline $1+2: 250-260 \mathrm{~cm}$ & Turtle & & plastron & 4 \\
\hline $3+4: 250-260 \mathrm{~cm}$ & Lizard ?species & & proximal humerus & 1 \\
\hline $3+4: 250-260 \mathrm{~cm}$ & Homo sapiens & human & proximal phalange-I/II & 1 \\
\hline NISP Total & & & & 252 \\
\hline
\end{tabular}


Most of the small collection of mammal bone was from human, with pig and flying fox in Layer 1 and the Pacific rat (Rattus exulans), only in Layer 4. A single rib fragment in Layer 4 was identified as belonging to a medium-sized mammal, possibly pig, but no unequivocal evidence for the mammalian commensals, pig and dog, was recorded from the pre-1000 BP deposit. Gifford (1951) recorded pig bone in his excavations, at a depth of about $2.6 \mathrm{~m}$. The human bone from earlier and recent excavations consisted of fragments from the cranium, mandible, arm, hand and leg. The remains do not appear to be from a disturbed primary or secondary burial. Analysis by David DeGusta (pers. comm., University of California) of human remains from the 1947 and 1996 excavations identified cut marks and modification (burning, ancient breaks, crushing, percussion pits), consistent with cannibalism (Figure 114), which supports Gifford's (1951:108) assessment that: 'it seems clear that human flesh was eaten in the Early period'. Redating of the Layer 4 deposit indicates that cannibalism was present in Fiji before 1000 BP (see also Best 1984:562, 592).

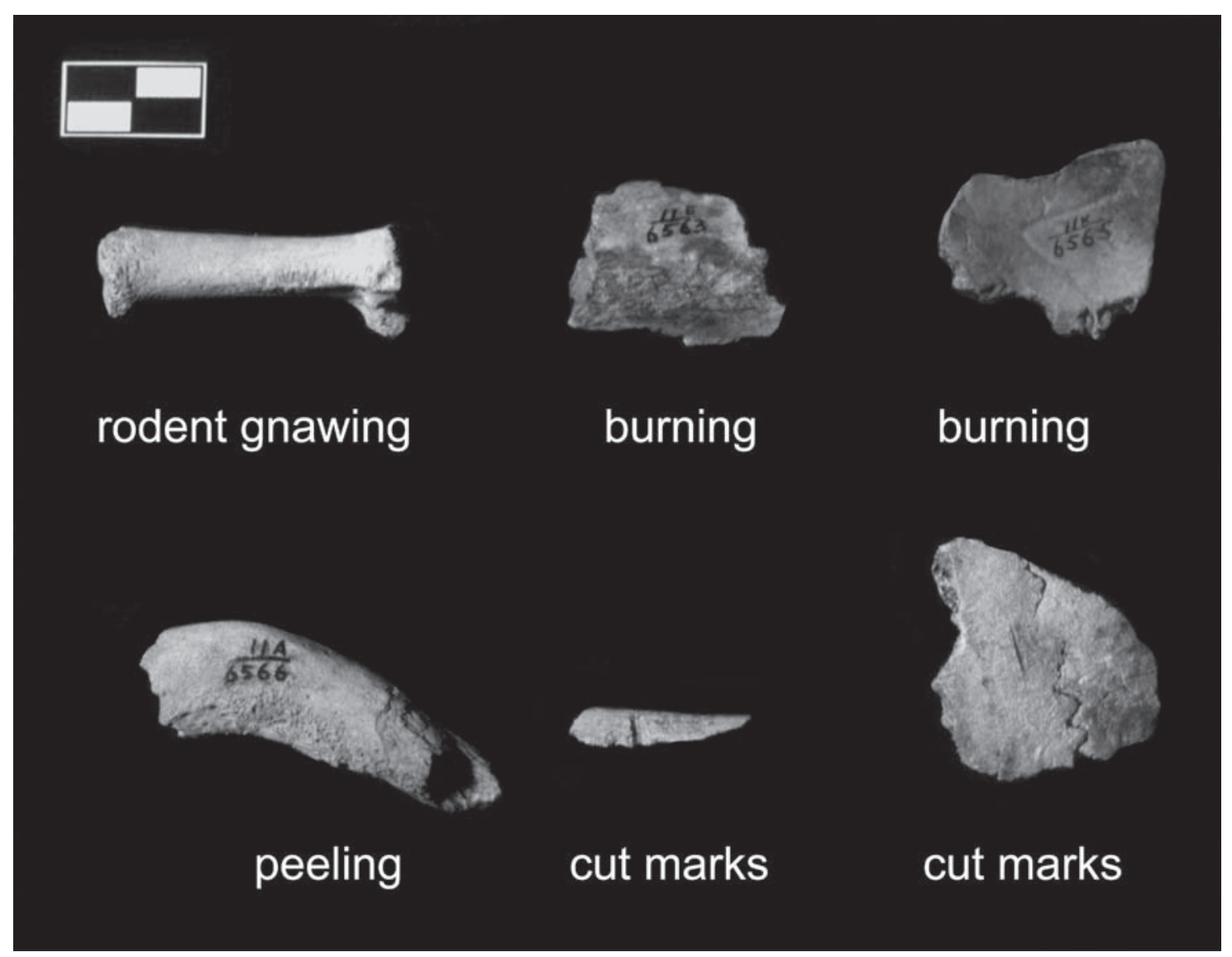

Figure 114. Modification of human bone at the Navatu 17A site. Photo courtesy of David DeGusta.

\section{Kulu Bay (Beqa Island)}

The non-fish fauna from Kulu contained remains from three domestic species (pig, dog and chicken), in addition to a large species and a small species of Rattus (Table 40). The large species had remains of comparable size to bones from $R$. praetor, and the smaller species was comparable in size to $R$. exulans. Kulu is one of the few Lapita deposits in Fiji that might contain the full suite of animal species that were transported to the Pacific in prehistory (see also Nunn et al. 2007). However, the Kulu sediments were water-transported and the deposit contains ceramics of post-Lapita age along with Lapita sherds. Without directly dating the remains of domestic 
Table 40. Kulu Bay non-fish remains.

\begin{tabular}{|c|c|c|c|c|}
\hline Provenience & Taxa & Common name & Element & NISP \\
\hline C9: $10-20 \mathrm{~cm}$ & Mammal ?species & & fragment vertebra & 1 \\
\hline C9: $10-20 \mathrm{~cm}$ & Mammal ?species & & metatarsal & 1 \\
\hline C9: $20-30 \mathrm{~cm}$ & Mammal ?species & & fragment & 1 \\
\hline C9 $30-40 \mathrm{~cm}$ & Turtle sp. & & plastron & 3 \\
\hline C9: $30-40 \mathrm{~cm}$ & Gallus gallus & Domestic chicken & proximal tarsometatarsus (L) & 1 \\
\hline C9: $30-40 \mathrm{~cm}$ & Sus scrofa & Pig & vertebra & 1 \\
\hline C9: $40-50 \mathrm{~cm}$ & Canis familiaris & Dog & tooth-incisor & 1 \\
\hline C9: $40-50 \mathrm{~cm}$ & Turtle sp. & & plastron & 3 \\
\hline C9: $40-50 \mathrm{~cm}$ & Bird ?species & & long bone fragment & 1 \\
\hline C9: 40-50 cm & Bird ?species & & long bone fragment & 1 \\
\hline C9: $50-60 \mathrm{~cm}$ & Turtle sp. & & plastron & 4 \\
\hline C9: $50-60 \mathrm{~cm}$ & Pteropus cf. tonganus & Large fruit bat & mandible & 1 \\
\hline (9: $50-60 \mathrm{~cm}$ & Bird?species & & fragment long bone & 3 \\
\hline $\mathrm{C} 10: 10-20 \mathrm{~cm}$ & Mammal ?species & & long bone fragment & 1 \\
\hline $\mathrm{C} 10: 30-50 \mathrm{~cm}$ & Mammal ?species & & fragment & 3 \\
\hline $\mathrm{C} 10: 50-70 \mathrm{~cm}$ & Turtle sp. & & plastron & 2 \\
\hline $\mathrm{C} 10: 60-80 \mathrm{~cm}$ & Canis familiaris & Dog & tooth-canine & 1 \\
\hline $\mathrm{C} 10: 60-80 \mathrm{~cm}$ & Gallus gallus & Domestic chicken & proximal tarsometatarsus (L) & 1 \\
\hline $\mathrm{C} 10: 70-80 \mathrm{~cm}$ & Mammal ?species & & fragment vertebra & 1 \\
\hline C10: $90-100 \mathrm{~cm}$ & Canis familiaris & Dog & tooth-incisor & 2 \\
\hline $\mathrm{C} 10: 90-100 \mathrm{~cm}$ & Mammal ?species & & fragment & 2 \\
\hline C10: $90-100 \mathrm{~cm}$ & Homo sapiens & Human & tooth-molar & 1 \\
\hline $\mathrm{C} 11: 0-10 \mathrm{~cm}$ & Mammal ?species & & long bone fragment & 1 \\
\hline $\mathrm{C} 11: 20-30 \mathrm{~cm}$ & Turtle sp. & & fragment & 2 \\
\hline $\mathrm{C} 11: 20-30 \mathrm{~cm}$ & Mammal ?species & & fragment & 1 \\
\hline $\mathrm{C} 11: 30-40 \mathrm{~cm}$ & Canis familiaris & Dog & tooth-premolar & 1 \\
\hline $\mathrm{C} 11: 30-40 \mathrm{~cm}$ & Rattus sp. & Rat & distal tibia & 1 \\
\hline $\mathrm{C} 11: 30-40 \mathrm{~cm}$ & Mammal ?species & & fragment & 5 \\
\hline $\mathrm{C} 11: 60-70 \mathrm{~cm}$ & Mammal ?species & & long bone fragment & 1 \\
\hline $\mathrm{C} 11: 70-80 \mathrm{~cm}$ & Mammal ?species & & fragment & 5 \\
\hline $\mathrm{C} 11: 70-80 \mathrm{~cm}$ & Rattus sp. & Rat & mandible (L) & 1 \\
\hline $\mathrm{C} 11: 70-80 \mathrm{~cm}$ & Sus scrofa & Pig & tooth-incisor & 1 \\
\hline $\mathrm{C} 11: 70-80 \mathrm{~cm}$ & Ducula cf. lakeba & Lau imperial pigeon & distal coracoid (R) & 1 \\
\hline $\mathrm{C} 11: 80-90 \mathrm{~cm}$ & Turtle sp. & & plastron & 3 \\
\hline $\mathrm{C} 11: 80-90 \mathrm{~cm}$ & Rattus sp. & Rat & humerus (R) & 1 \\
\hline C11: 80-90 cm & Mammal ?species & & fragment & 7 \\
\hline $\mathrm{C} 11: 80-90 \mathrm{~cm}$ & cf. Puffinus pacificus & Wedge-tailed shearwater & shaft humerus (R) & 1 \\
\hline C11: $90-100 \mathrm{~cm}$ & Rattus sp. & Rat & vertebra & 1 \\
\hline C11: $90-100 \mathrm{~cm}$ & Canis familiaris & Dog & tooth-incisor & 2 \\
\hline C11: $90-100 \mathrm{~cm}$ & Sus scrofa & Pig & tooth-incisor & 1 \\
\hline C11: $90-100 \mathrm{~cm}$ & Mammal ?species & & fragment & 6 \\
\hline C11: $100-110 \mathrm{~cm}$ & Turtle sp. & & fragment & 4 \\
\hline C11: $100-110 \mathrm{~cm}$ & Rattus sp. & Rat & shaft humerus & 1 \\
\hline C11: $100-110 \mathrm{~cm}$ & Rattus sp. & Rat & shaft tibia & 1 \\
\hline C11: $100-110 \mathrm{~cm}$ & Rattus sp. & Rat & pelvis (L) & 1 \\
\hline
\end{tabular}


Table 40 continued

\begin{tabular}{|c|c|c|c|c|}
\hline Provenience & Taxa & Common name & Element & NISP \\
\hline C11: 100-110 cm & Mammal ?species & & fragment & 4 \\
\hline C11: $110-120 \mathrm{~cm}$ & Canis familiaris & Dog & tooth-incisor & 1 \\
\hline C11: 110-120 cm & Sus scrofa & Pig & tooth-incisor & 1 \\
\hline C11: $110-120 \mathrm{~cm}$ & Rattus sp. & Rat & femur (R) & 2 \\
\hline C11: $110-120 \mathrm{~cm}$ & Rattus sp. & Rat & femur (L) & 1 \\
\hline C11: $110-120 \mathrm{~cm}$ & Rattus sp. & Rat & tibia & 2 \\
\hline C11: $110-120 \mathrm{~cm}$ & Rattus sp. & Rat & humerus (R) & 1 \\
\hline C11: $110-120 \mathrm{~cm}$ & Rattus sp. & Rat & pelvis (R) & 2 \\
\hline C11: $110-120 \mathrm{~cm}$ & Rattus sp. & Rat & pelvis & 1 \\
\hline C11: $110-120 \mathrm{~cm}$ & Rattus sp. & Rat & caudal vertebra & 2 \\
\hline C11: $110-120 \mathrm{~cm}$ & Mammal ?species & & vertebra & 1 \\
\hline C11: $110-120 \mathrm{~cm}$ & Mammal ?species & & fragment & 11 \\
\hline C11: $110-120 \mathrm{~cm}$ & Bird ?species & & fragment long bone & 1 \\
\hline C11: $120-130 \mathrm{~cm}$ & Rattus sp. & Rat & humerus (R) & 1 \\
\hline C11: $120-130 \mathrm{~cm}$ & Canis familiaris & Dog & tooth-incisor & 1 \\
\hline C11: $120-130 \mathrm{~cm}$ & Mammal ?species & & fragment & 2 \\
\hline C11: $130-140 \mathrm{~cm}$ & Homosapiens & Human & tooth-molar fragment & 1 \\
\hline C11: 130-140 cm & Canis familiaris & Dog & tooth-incisor & 1 \\
\hline C11: $130-140 \mathrm{~cm}$ & Mammal ?species & & long bone fragment & 1 \\
\hline C11: 130-140 cm & Bird ?species & & fragment long bone & 1 \\
\hline C11: 150-160 cm & Mammal ?species & & long bone fragment & 2 \\
\hline NISP Total & & & & 125 \\
\hline
\end{tabular}

species, we cannot be certain that the Lapita occupation at Kulu involved the husbandry of introduced animals. Only a few identifiable bird bones were recovered from the excavations, with remains of Gallus gallus and a Procellarid, probably the wedge-tailed shearwater. Shearwaters are medium-sized pelagic birds that nest mostly in ground burrows and are mainly nocturnal on their breeding grounds (Pratt et al. 1987:51). Interestingly, the middle levels of the site had a single bone from the extinct volant pigeon (Ducula lakeba) that is otherwise known from the early-Lapita deposits on Lakeba Island, and the pigeon probably existed on many Fiji Islands in the past (Worthy 2001b; Steadman 2006a). The small amount of human bone did not exhibit any evidence of cannibalism and may represent a redeposited early burial.

\section{Volivoli II, Volivoli III and Malaqereqere (south Viti Levu)}

Volivoli II

The fauna from Volivoli II consisted mainly of bones from Rattus sp., along with sparse amounts of bird, turtle, fruit bat, pig and goat bone (Table 41). The small amount of bird bone is significant in that it contains bones from the small collared petrel (Pterodroma brevipes), which might have bred in large numbers on the inland hills of Viti Levu before human activity and the arrival of introduced predators such as rats.

\section{Volivoli III}

The site contained remains of the fruit bat and unidentified gecko and bird species (Table 41). 


\section{Malaqereqere}

In contrast to the numerous fish bones in the coastal rock shelter, the non-fish bone component included sparse remains from fruit bat and a Rattus sp., probably $R$. exulans. An unidentified rail (cf. Gallirallus philippensis) was present, along with two reptiles (Table 41). One of these is the Pacific boa (Candoia sp.), which is currently widespread in Fiji, while the other is the extinct giant ground frog (Platymantis megabotoniviti) that is assumed to have become extinct after the arrival of people and rodents to Fiji (Worthy 2001a).

Table 41. Volivoli Il, Volivoli III and Malaqereqere non-fish remains.

\begin{tabular}{|c|c|c|c|c|}
\hline Provenience & Taxa & Common name & Element & NISP \\
\hline $0-10 \mathrm{~cm}$ & Susscrofa & Pig & tooth-incisor & 3 \\
\hline $0-10 \mathrm{~cm}$ & Susscrofa & Pig & phalange & 1 \\
\hline $0-10 \mathrm{~cm}$ & Mammal ?species & & long bone fragments & 22 \\
\hline $10-20 \mathrm{~cm}$ & Caprahircus & Domestic goat & tooth-molar & 1 \\
\hline $10-20 \mathrm{~cm}$ & Rattus sp. & Rat & tibia (R) & 1 \\
\hline $10-20 \mathrm{~cm}$ & ?Species & & fragments & 6 \\
\hline $20-30 \mathrm{~cm}$ & Turtle sp. & & plastron & 33 \\
\hline $20-30 \mathrm{~cm}$ & Rattus sp. & Rat & mandible (R) & 2 \\
\hline $20-30$ & Rattus sp. & Rat & tooth-incisor & 1 \\
\hline $20-30$ & Rattus sp. & Rat & femur (L) & 7 \\
\hline $20-30$ & Rattus sp. & Rat & femur (R) & 4 \\
\hline $20-30$ & Rattus sp. & Rat & ulna (R) & 2 \\
\hline $20-30$ & Rattus sp. & Rat & humerus (L) & 1 \\
\hline $20-30$ & Rattus sp. & Rat & humerus (R) & 1 \\
\hline $20-30$ & Rattus sp. & Rat & pelvis (R) & 1 \\
\hline $20-30$ & Rattus sp. & Rat & tibia (R) & 5 \\
\hline $20-30$ & Rattus sp. & Rat & tibia (L) & 2 \\
\hline $20-30$ & Gallirallus philippensis & Banded rail & proximal humerus (R) & 1 \\
\hline $20-30$ & ?Species & & fragments & 9 \\
\hline $30-40$ & Rattus sp. & Rat & femur (L) & 12 \\
\hline $30-40$ & Rattus sp. & Rat & femur (R) & 6 \\
\hline $30-40$ & Rattus sp. & Rat & humerus (L) & 1 \\
\hline $30-40$ & Rattus sp. & Rat & humerus (R) & 1 \\
\hline $30-40$ & Rattus sp. & Rat & vertebrae & 1 \\
\hline $30-40$ & Rattus sp. & Rat & mandible (R) & 3 \\
\hline $30-40$ & Rattus sp. & Rat & mandible (L) & 1 \\
\hline $30-40$ & Rattus sp. & Rat & maxilla (R) & 1 \\
\hline $30-40$ & Rattus sp. & Rat & pelvis (R) & 2 \\
\hline $30-40$ & Rattus sp. & Rat & pelvis $(\mathrm{L})$ & 3 \\
\hline $30-40$ & Rattus sp. & Rat & tibia (L) & 5 \\
\hline $30-40$ & Rattus sp. & Rat & tibia (R) & 7 \\
\hline $30-40$ & Pterodrama cf. brevipes & Small collared petrel & proximal humerus $(\mathrm{L})$ & 1 \\
\hline $30-40$ & ?Species & & fragments & 7 \\
\hline $40-50$ & Turtle sp. & Turtle & plastron & 7 \\
\hline $40-50$ & Rattus sp. & Rat & tibia (L) & 4 \\
\hline
\end{tabular}


248 Trevor H. Worthy and Geoffrey Clark

Table 41 continued

\begin{tabular}{|c|c|c|c|c|}
\hline Provenience & Taxa & Common name & Element & NISP \\
\hline $40-50$ & Rattus sp. & Rat & tibia (R) & 2 \\
\hline $40-50$ & Rattus sp. & Rat & femur (R) & 4 \\
\hline $40-50$ & Rattus sp. & Rat & femur (L) & 3 \\
\hline $40-50$ & Rattus sp. & Rat & humerus (L) & 2 \\
\hline $40-50$ & Rattus sp. & Rat & ulna (L) & 1 \\
\hline $40-50$ & Pterodrama cf. brevipes & Small collared petrel & distal humerus $(L)$, radius & 2 \\
\hline $40-50$ & ?Species & & fragments & 16 \\
\hline $50-60$ & Turtle sp. & Turtle & plastron & 2 \\
\hline $50-60$ & Rattus sp. & Rat & mandible (L) & 1 \\
\hline $50-60$ & Rattus sp. & Rat & mandible (R) & 1 \\
\hline $50-60$ & Rattus sp. & Rat & pelvis (?) & 1 \\
\hline $50-60$ & Rattus sp. & Rat & tibia (L) & 1 \\
\hline $50-60$ & Rattus sp. & Rat & scapula & 1 \\
\hline $50-60$ & ?Species & & fragments & 7 \\
\hline $60-70$ & Rattus sp. & Rat & humerus (L) & 1 \\
\hline $60-70$ & ?Species & & long bone fragments & 1 \\
\hline Posthole fill & ?Species & & fragments & 2 \\
\hline Posthole fill & ?Species & & tooth & 1 \\
\hline NISP Total & & & & 214 \\
\hline \multicolumn{5}{|l|}{ Volivoli III } \\
\hline Spit V & Pteropus cf. tonganus & Large fruit bat & mandible & 1 \\
\hline Spit VIII & Pteropus cf. tonganus & Large fruit bat & long bones & 4 \\
\hline Spit VIII & Gecko sp. & Gecko & fragment & 1 \\
\hline Spit VIII & Bird ?species & & long bone & 1 \\
\hline NISP Total & & & & 7 \\
\hline \multicolumn{5}{|l|}{ Malaqereqere } \\
\hline A1: $10-20 \mathrm{~cm}$ & Pteropus cf. tonganus & Large fruit bat & long bones & 5 \\
\hline $\mathrm{A} 1: 20-30 \mathrm{~cm}$ & Pteropus cf. tonganus & Large fruit bat & long bones & 4 \\
\hline $\mathrm{A} 1: 30-40 \mathrm{~cm}$ & Pteropus cf. tonganus & Large fruit bat & long bones & 5 \\
\hline $\mathrm{A} 1: 30-40 \mathrm{~cm}$ & Rattus sp. & Rat & long bone & 1 \\
\hline $\mathrm{A} 1: 30-40 \mathrm{~cm}$ & Candoia bibroni & Pacific boa & vertebra & 1 \\
\hline $\mathrm{A} 1: 40-50 \mathrm{~cm}$ & Pteropus cf. tonganus & Large fruit bat & long bone & 1 \\
\hline $\mathrm{A} 1: 40-50 \mathrm{~cm}$ & Rattus sp. & Rat & long bone, pelvis & 1 \\
\hline A2: $10-20 \mathrm{~cm}$ & Pteropus cf. tonganus & Large fruit bat & long bones & 5 \\
\hline A2: $10-20 \mathrm{~cm}$ & Rail ?species & Rail & proximal tarsometatarsus & 1 \\
\hline$A 2: 50-60 \mathrm{~cm}$ & Porphyrio porphyrio & Purple swamphen & shaft tibiotarsus (R) & 1 \\
\hline$A 2: 50-60 \mathrm{~cm}$ & Platymantis megabotoniviti & Giant Fiji ground frog & vertebra & 1 \\
\hline$A 2: 50-60 \mathrm{~cm}$ & Rattus sp. & Rat & long bones & 2 \\
\hline NISP Total & & & & 28 \\
\hline
\end{tabular}




\section{Qaranioso II and Tuvu (west Viti Levu)}

\section{Qaranioso II}

The inland shelter produced small amounts of pottery and faunal material (Table 42), which is significant for two reasons. First, a decorated ceramic rim of late-Lapita age showed that people were using the interior regions of Viti Levu early in prehistory and affecting the environment (Anderson et al. 2000). Evidence for this at Qaranioso II is suggested by the remains of a large extinct iguana (Lapitiguana impensa). This iguana had an estimated snout-to-vent length of 500 $\mathrm{mm}$ (Pregill and Worthy 2003), and it was much larger than either of the three iguanas in Fiji today (Brachylophus fasciatus, B. bulabula and B. vitiensis). In Tonga, bones from a large iguana (Brachylophus gibbonsi) have been found in several Lapita sites and direct radiocarbon dating of iguana bones suggests it became extinct there within a century or two of human arrival (Steadman et al. 2002). Although radiocarbon dating of the iguana bone from Qaranioso II failed due to a lack of adequate dateable material, the co-occurrence of the remains of Lapitiguana impensa with pottery of late-Lapita age suggests a similar pattern of human predation to that witnessed on Tonga. The rock shelter also contained bat remains (microbat, Notopterus macdonaldi), Pacific boa and banded rail, as well as three species of Rattus ( $R$. exulans, $R$. praetor, $R$. rattus). The ratbone sequence includes $R$. praetor bone found in the $10-20 \mathrm{~cm}$ level of the shelter, while the

Table 42. Qaranioso II and Tuvu non-fish remains.

\begin{tabular}{|c|c|c|c|c|}
\hline Provenience & Taxa & Common name & Element & NISP \\
\hline \multicolumn{5}{|l|}{ Qaranioso II } \\
\hline TP 1: 0-10 cm & Gallirallus philippensis & Banded rail & long bone & 1 \\
\hline TP 1: 0-10 cm & Rattus cf. rattus & Black rat & long bones & 6 \\
\hline TP 1: 0-10 cm & Rattus cf. exulans & Pacific rat & long bone & 1 \\
\hline TP 1: 10-20 cm & Rattus exulans & Pacific rat & long bones & 4 \\
\hline TP 1: $10-20 \mathrm{~cm}$ & Rattus sp. (large) & Rat & long bones & 5 \\
\hline TP 1: 10-20 cm & Rattus praetor & Spiny rat & mandible & 1 \\
\hline TP 1: 10-20 cm & Gallus sp. & & proximal radius, phalange & 2 \\
\hline TP 1: 10-20 cm & Microchiroptera & Microbat & long bone & 1 \\
\hline TP 1: 20-30 cm & Rattus praetor & Spiny rat & maxilla & 1 \\
\hline TP $1: 40-50 \mathrm{~cm}$ & Bird ?species & & fragments & 2 \\
\hline $\mathrm{TP}: 50-60 \mathrm{~cm}$ & Notopteris macdonaldi & Long-tailed fruit bat & & 11 \\
\hline TP 1: 50-60 cm & Rattus cf exulans & Pacific rat & long bones & 2 \\
\hline TP 1: 50-60 cm & Candoia cf. bibroni & Pacific boa & vertebra & 3 \\
\hline TP 1: 50-60 cm & ?Platymantis vitianus & Fiji ground frog & fragment & 1 \\
\hline TP 1: 50-60 cm & Bird ?species & & fragment & 1 \\
\hline TP 1: $60-70 \mathrm{~cm}$ & Lapitiguana impensa & Fiji giant iguana & shaft scapulacorocoid (R), chevron bone & 2 \\
\hline TP 1: 60-70 cm & Rattus sp. (large) & Rat & long bone & 1 \\
\hline NISP Total & & & & 45 \\
\hline \multicolumn{5}{|l|}{ Tuvu } \\
\hline Spit 3 & Rattus sp. & Rat & tibia & 1 \\
\hline
\end{tabular}


European-introduced $R$. rattus was found above it, in the top $0-10 \mathrm{~cm}$ level. A radiocarbon date from $30 \mathrm{~cm}$ depth has an age of 530-670 cal. BP (ANU-11014), indicating that $R$. praetor survived until recent times and became extinct after the arrival of European species of Rattus.

\section{Tuvu}

A single bone of Rattus sp. was recovered from the Tuvu deposit (Table 42).

\section{Discussion}

The prehistoric fauna from archaeological sites in Fiji includes several extinct species found in deposits containing material culture of Lapita age. Two extinct reptiles and a frog - the giant iguana (Lapitiguana impensa), endemic crocodilian (Volia athollandersoni) and giant ground frog (Platymantis megabotoniviti) - have all been found on Viti Levu or on nearby islands, but not as yet in the Lau Group. Extinct reptile remains were found in cultural deposits at Qaranioso II and Malaqereqere, but whether the reptile remains result from human predation or natural deposition is unclear. Bone from turtle and fruit bat is found in small amounts in many Lapita and post-Lapita sites, with the Qaranipuqa Lapita levels having greater amounts of both than post-Lapita levels. Extinct land birds have been identified at sites with Lapita ceramics, such as Kulu (Beqa Island), Votua (Mago Island), Qaranipuqa (Lakeba Island) and Naigani Island, but not in a late-Lapita deposit on Waya Island (Steadman 2006a:166, Tables 6-8). A site on Aiwa Levu Island (ALR1) in the Lau Group with extinct bird bone appears to contain avifauna from pre-human deposits, as well as those of post-Lapita age. The association of the extinct species with the post-Lapita cultural deposit is unclear (Steadman 2006a:178).

Elsewhere in Fiji, bone from extinct bird species, however, is absent from post-Lapita deposits on Viti Levu (e.g. Sigatoka, Navatu, Malaqereqere, Volivoli II) and the Lau Group (Aiwa Lailai Island, Nayau Island, Lakeba Island) (Steadman 2006a). This suggests that Fiji's avifauna, especially its endemic species, was significantly depleted during the first centuries of Lapita occupation. The effect of human arrival on Fiji's native birds is most clearly seen at the Lakeba site of Qaranipuqa excavated by Simon Best in the 1970s, and the bird remains are discussed further below.

\section{The Lakeba avifauna sequence}

At least 26 native species of birds are represented in the fauna from Qaranipuqa and Wakea. A striking feature of the fauna is that it is dominated by land birds and poorly represents sea birds. For example, petrels, terns, and boobies, which are prominent in the extant fauna of the region today (Watling 1982), are entirely lacking. Both groups are often very common in tropical Pacific archaeological sites (Steadman 1995, 2006a), e.g. on Niue (Worthy et al. 1998), American Samoa (Steadman 1994), Mangaia (Steadman 1985; Steadman and Kirch 1990), and Henderson Island (Wragg and Weisler 1994; Wragg 1995). It may be that these colonial nesting birds were not available in the near vicinity of Qaranipuqa, as those sea birds that were taken would have been available nesting in the forest (e.g. white-tailed tropicbird) or on the adjacent coast as individuals (e.g. the herons and godwit).

When the fauna is analysed by depth, as in Table 37, it is obvious most of the fauna came from the lower layers $\mathrm{T}-\mathrm{X}$, with a lesser accumulation in $\mathrm{H}-\mathrm{O}$, and scattered bones in A-F. Layers A-F date largely to the past 1000 years, and there is a discontinuity in the Qaranipuqa deposits between Layers A-F and underlying layers (Best 1984:Table 2.2).

It is likely that all fauna from Layers A and B, at least, and possibly as deep as Layer F, is derived, not from cultural activity, but from the middens of barn owls (Tyto alba) that lived 
in the shelter. All of the species recorded in these upper layers would be able to be taken by barn owls. Faunas from modern barn-owl middens on Viti Levu and Vatulele (see Chapter 3) indicate that barn owls regularly take Egretta and shore birds when available, in addition to small forest birds. Therefore, in the Qaranipuqa fauna, both waders and the heron are almost certainly owl-caught species. Similarly, the small passerines, the kingfisher and the lorikeet bones are also probably from the owl midden.

The fauna from the middle part of the sequence (Layers $\mathrm{H}-\mathrm{O}$ ) represents a comparatively depauperate fauna being taken by the human inhabitants of Qaranipuqa. These layers are radiocarbon dated to between about $2300 \mathrm{cal}$. BP (Layer O) and $1800 \mathrm{cal}$. BP (Layer H). The hunted species were mainly rails, a few pigeons and a few sea birds (white-tailed tropicbird and frigate bird). In contrast, the Lapita people, represented in the earliest layers, $\mathrm{T}-\mathrm{X}$, hunted a diverse range of species that included no sea birds.

The Qaranipuqa fauna reveals that the following taxa have gone extinct on Lakeba, and did so during deposition of the lower layers of the site, as none occurs above Lapita horizons: the megapode Megapodius alimentum (as M. freycinet in Best 1984), a large pigeon (Ducula lakeba), the tooth-billed pigeon (Didunculus strigirostris), and the musk parrot (Prosopeia sp.). Porphyrio porphyrio has since become extinct on the island (Steadman and Franklin 2000).

Several taxa await reference material for certain identification. For example, the lorikeet Charmosyma amabilis is probably represented in the Lakeba fauna, which would be a significant extension of its present range in Viti Levu, Taveuni and Ovalau (Watling 1982). Several pigeon bones bigger than Ducula pacifica and smaller than the large extinct Ducula lakeba also await more extensive reference material for certain identification, but are likely to be Ducula latrans. This large-bodied fruit pigeon is currently restricted to the large Fiji Islands and its range includes Lakeba (Watling 1982). One tarsometatarsus represents a Ptilinopus sp. that is larger than any of the species of Ptilinopus extant in Fiji. However, both $P$. rarotongensis of Rarotonga and $P$. purpuratus of the Society Islands-Tuamotu Group have similar-sized tarsometatarsi (Steadman 1992). The fossil may indicate that a dove larger than those in the golden dove group or $P$. porphyraceus and $P$. perousii and similar in size to these more eastern taxa formerly lived on the Lau Group.

Of the extinct pigeons, Ducula lakeba has not yet been recorded from elsewhere, although a similar-sized pigeon (perhaps the same taxon) is known from Viti Levu and Beqa (Worthy 2001b). Ducula lakeba and D. david (Balouet and Olson 1987) are the largest fruit pigeons described from the Pacific, and are only a little smaller than the large New Zealand Hemiphaga novaeseelandiae. Steadman $(1989,1997)$ recorded Ducula david from Foa and Lifuka in the Ha'apai Group and 'Eua, all in the Kingdom of Tonga, and provisionally lists this species from Lakeba (Steadman 1997). The species D. david was based on a partial tarsometatarsus, and the only described Tongan bone, a coracoid, was referred by Steadman (1989) to D. david, based on its size. Steadman (1997) did not mention whether comparable material is included in the other sites. Steadman (1997) also listed a Ducula new sp. from Lifuka and 'Eua, which is presumed to be the same as that described in Steadman (1989) and as such is a much larger pigeon. However, following the distinction of D. lakeba from D. david (Worthy 2001b), the Tongan large Ducula bones were listed as an undescribed species (Steadman 2006a). The tooth-billed pigeon Didunculus strigirostris survives only in American Samoa, so the certain identification of several bones from Lakeba is a major extension of its range. Steadman $(1993,1997)$ reported a new species of Didunculus from two caves on 'Eua. It has recently been named (Steadman 2006c).

The presence of a megapode on Lakeba and in Votua on Mago (see Clark et al. 2001) elsewhere in the Lau Group is not unexpected as various species have been reported previously 
from island groups to the east of their main extant distribution in the western Pacific (Jones et al. 1995), as far as Niue (Steadman et al. 2000). At least four species of megapodes are known from Tonga (Steadman 1999, 2006a). Of these, the medium-sized scrubfowl M. alimentum (Steadman 1989) is here confirmed as that from Lakeba, as suggested by Steadman (1999). This species is also reported from Mago Island, but contra Steadman (1999), it has not been found on Naigani off Viti Levu. That megapode was the very large Megavitiornis altirostris (Worthy 2000). Megapodius alimentum was a volant species and so was probably widespread in the Lau Group and east to Tonga. On Viti Levu and probably on Vanua Levu, however, the larger and probably flightless Megapodius amissus (Worthy 2000) replaced M. alimentum.

The age of the older Lapita fauna from Qaranipuqa (Layer W to Layer R/S) has been reevaluated by Anderson and Clark (1999) as about 2700-2900 cal BP. It is within this period that the extinctions occurred. The composition of the original fauna included the black duck Anas superciliosa, which is still widespread in the Pacific and common on the island today (Steadman and Franklin 2000), occupying both freshwater wetlands and coastal habitats. Of the rails, Porphyrio porphyrio was present in the early and middle parts of the sequence and has probably gone extinct historically as it has elsewhere in the Fiji archipelago following the impact of mammalian predation. The banded rail Gallirallus philippensis may have been a more recent arrival, as all bones were only recorded from upper horizons. Perhaps either habitat changes or faunal extinctions after human arrival enabled the banded rail to establish populations on Lakeba. The revised identification confirms the presence of Gallus gallus in the Lapita levels of the site, although secure evidence of the pig and dog in sites of Lapita age in Fiji and Tonga has yet to be recovered (Burley 1998; Clark and Anderson 2001).

All the Tyto bones come from upper layers in the site, conforming with a suggested posthuman expansion by this owl into the Pacific (see Chapter 3) following the introduction of rodents. There are no prehuman records of these owls known from Fiji (data herein), Niue (Worthy et al. 1998), or elsewhere in Polynesia (Steadman 2006a), so far as the authors are aware.

The modern fauna of Lakeba includes 21 land and freshwater birds (Steadman and Franklin 2000). Their survey shows that both Gallirallus philippensis and Gallicolumba stairii are present, although rare, due probably to the presence of abundant feral cats. The pigeons include the two doves Ptilinopus porphyraceus and $P$. perousii, in addition to the fruit pigeons $D$. pacifica and $C$. vitiensis. The modern fauna contains several species not found in the archaeological fauna: Circus approximans harrier, Collocalia spodiopygia swiftlet, and the small passerines Lalage maculosa, Mayrornis lessoni, Myiagra vanikorensis and Myzomela jugalis. These six species plus P. perousii are likely to have been former inhabitants of Lakeba and so add to the 20 land and freshwater species in the archaeological fauna for a total of at least 27 land and freshwater species ( 33 in total) in the original fauna. This fauna is likely to be extended by the addition of extinct flightless rails such as have been found in the Cook Islands (Steadman 1987) and Niue (Steadman et al. 2000).

\section{Commensal introduction and post-Lapita subsistence}

The extinction of land birds and probably several taxa of reptile appears to have taken place during the Lapita phase in Fiji and involved heavy human predation in the case of land birds. The impact that commensal species had on the demise of Fijis native fauna during the colonisation era is unclear because a Lapita association for the three domesticates, pig, dog and chicken, and two species of Rattus ( $R$. praetor and $R$. exulans) in disturbed sites is questionable. This is an important issue since domestic animals often serve as a proxy marker of a horticultural society, 
and if a transported economic system of flora and fauna underwrote, to some extent, Lapita subsistence, then human impact on Fiji's indigenous taxa might have, in the short term, been less harmful, and secondary to the negative effects of introduced commensal species. Conversely, an absence of commensal species in Lapita times could favour a scenario involving the colonists' early reliance on Fiji's wild foods, with the subsequent introduction of domesticates and the increased probability of additional migrants arriving in Fiji during the Lapita era.

The two species of Rattus introduced to Fiji in prehistory ( $R$. exulans and $R$. praetor) both appear to be early introductions and have been found in several Lapita sites, such as Votua and Qaranipuqa in the Lau Group, and on Viti Levu, although $R$. praetor has not so far been reported from the Yasawas or Aiwa Islands (Cochrane 2006; Jones et al. 2007). At the Qaranipuqa site on Lakeba, both $R$. praetor and $R$. exulans were found together in Layers $\mathrm{P}-\mathrm{T}$, which overlie Layers U-X (White et al. 2000) that contain abundant remains of extinct land birds, especially Megapodius alimentum and Ducula lakeba. It is feasible that the two species of Rattus reached the Lau Group shortly after initial human arrival. The geographic distribution of $R$. praetor is intriguing, as it was evidently introduced to Vanuatu, but is not recorded from Lapita sites in New Caledonia or Tonga (White et al. 2000; Grant-Mackie et al. 2003). Attempts to establish the dispersal chronology of $R$. praetor by AMS dating its remains from New Ireland (Buang Merabak), Vanuatu (Navaprah) and Fiji (Votua and Sovanibeka) failed, with only one AMS determination able to be made on a $R$. praetor bone from the Sovanibeka deposit, with an age of 670-770 cal. BP (OZF882). The remains of $R$. praetor were common in the 'Navatu' levels at the Sigatoka Sand Dune on Viti Levu by 1340-1275 cal. BP (Burley 2005) and were found in upper levels of the Qaranioso II site, which indicates that $R$. praetor was sympatric with $R$. exulans for some time before becoming extinct in Fiji. The arrival of European rats probably caused the demise of $R$. praetor in Vanuatu and Fiji.

Like the two species of Rattus, the domestic fowl (Gallus gallus) was evidently brought to Fiji in Lapita times, with a single Gallus gallus bone in Layer W of the Qaranipuqa site and two chicken bones from Aiwa Levu and Aiwa Lailai that are AMS dated to 2000-2500 cal. BP, confirming its early presence in the Lau Group (Jones et al. 2007). The rarity of chicken bone from Lapita and post-Lapita sites in Fiji (Thomas et al. 2004; Burley 2005; Cochrane 2006; Jones et al. 2007; Nunn et al. 2007) contrasts with its frequency in archaeological deposits in Tonga, Niue and Rapa Nui, suggesting that Gallus gallus may have been semi-domesticated in Fiji, with feral populations present on many islands.

Both pig and dog have been recorded from mixed Lapita sites (e.g. Nunn et al. 2007) and occur at Kulu where the sediments have been redeposited. The restudy of suspected pig and dog remains from Yanuca and Naigani (Hunt 1980; Best 1981) has not been able to confirm their presence at these sites (T. Worthy pers. obs.; Figure 115). In well-preserved Lapita deposits such as Votua these species do not occur, and at the Qaranipuqa site they are found only in the uppermost layers (A-E1) dating to the last 1000 years, which is also the case for the pig at Navatu 17A (1996 excavations). In the Yasawas, there is pig bone from Level 15 of Qaranicagi (Site Y2-39), which has an estimated age range of 1000-2500 cal. BP (Cochrane 2006), while rare pig and dog bone is recorded from 'Navatu' and 'Plainware' levels at Sigatoka dated to ca. 1280-1520 cal. BP (Burley 2005). The age of the pig and dog remains found at two sites on Aiwa Levu and Aiwa Lailai is not reported (Jones et al. 2007). Thus, it is still uncertain when the pig and dog were introduced to Fiji, and whether these species had a major role in the decline of native species in Fiji. However, the relative absence of pig and dog remains in early archaeological sites suggests these animals played a minor role in Lapita subsistence, particularly in east Fiji. This also appears to be the case in Tonga and in New Caledonia where pigs seem 


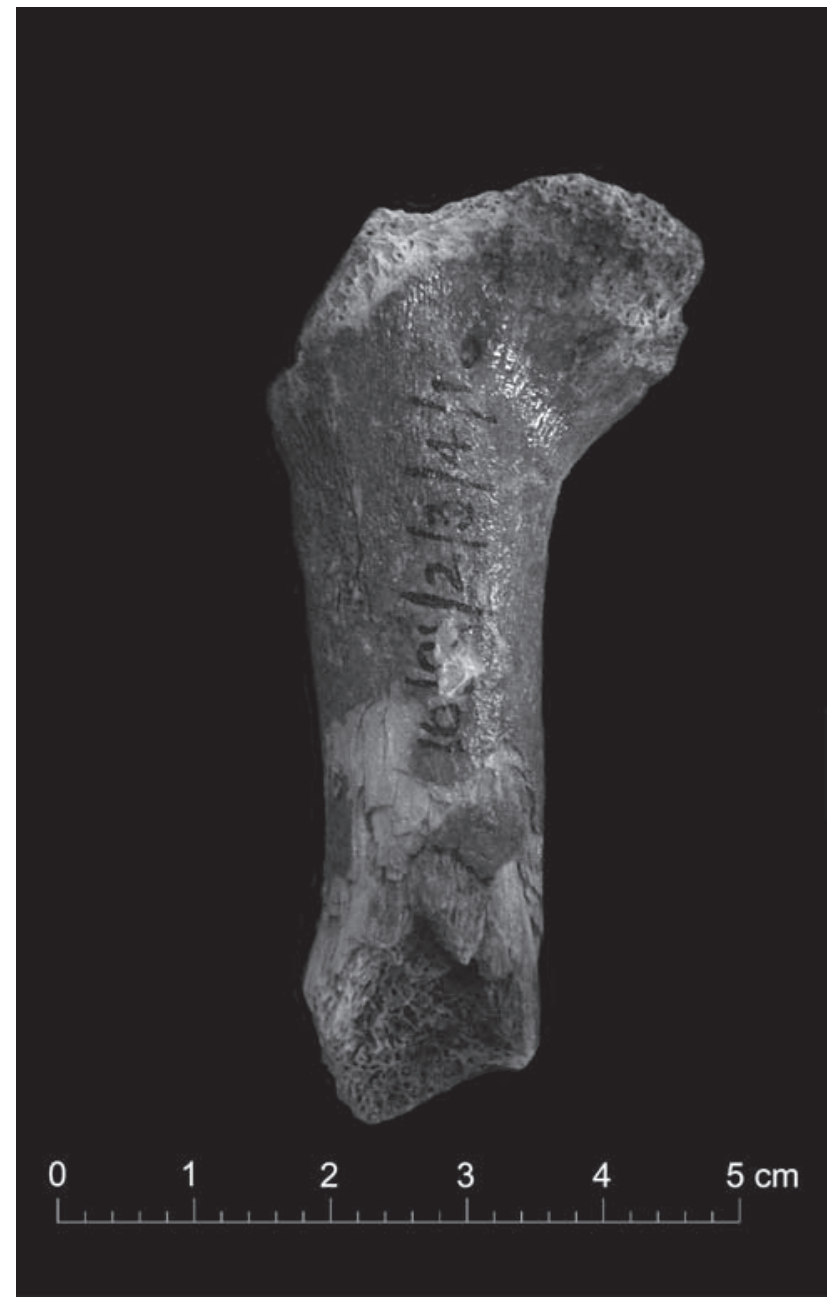

Figure 115. A small'pig' femur found in the Lapita levels at the Yanuca site (Trench 2/Zone 3/ Spit 4, see Hunt 1980:185-188) was re-examined by T.H. Worthy (University of New South Wales) and E.S. Gaffney (American Museum of Natural History) who identified it as turtle (Cheloniiade).

not to have been introduced in prehistory, but contrasts with some parts of Vanuatu where significant amounts of pig bone occurs in Lapita and early post-Lapita contexts. In Fiji, it is feasible that pigs were introduced to west Fiji, and were not initially taken to east Fiji. Direct dating of pig and dog bone from sites such as Naitabale and Bourewa (Nunn 2007; Nunn et al. 2007) is required to establish whether these animals were Lapita introductions to west Fiji.

If pig and dog had a limited role in Lapita subsistence in Fiji, as the current and admittedly scarce data suggests, then terrestrial sources of protein such as endemic reptiles and land bird taxa would likely have been sought during the colonisation era, leading to the extinction of vulnerable taxa in many parts of the archipelago within 200 to 300 years. The decline in native fauna would have necessitated a change in subsistence behaviour, particularly in foraging mobility in the immediate post-Lapita period, but archaeological sites and faunal assemblages from this period with which to evaluate this proposition are currently inadequate. Mobility was clearly greater during the colonisation phase than afterward, as shown by the evidence for significant early interaction on Lakeba (Best 1984; Green 1996; Clark 2000), but it is unclear whether early mobility relates, in part, to the ongoing exploitation of native fauna, or was primarily directed towards social interaction.

It has been argued that during the first millennium $\mathrm{AD}$ food stress is seen in the 'Navatu' faunal assemblage from Sigatoka that contains small fish and shellfish, along with Pacific boa, iguana and lizard, and several human remains from the burial area have enamel hypoplasia, possibly the result of food stress (Burley 2005). However, Pietrusewsky et al. (1994:32) note that 
in the Sigatoka human remains: 'overall very few teeth $(20 / 885$, or $2.3 \%)$ exhibit hypoplastic defects', suggesting the majority of Sigatoka people during the 'Fijian Plainware-Navatu' period did not suffer food stress.

Evidence for increasing inter-group competition from the occupation of naturally defended hilltops by 1500 BP has been proposed in the Sigatoka Valley by Field (2004), and cannibalism is evident from Navatu 17A by ca. 1400 BP, Vuda at ca. 1000 BP (DeGusta 2000), Qaranicagi (Y2-39) at ca. 750 BP (Cochrane et al. 2004), and Waya Island at ca. 500 BP (Rechtman 1992). On Lakeba, Best (1984:534-535) identified scattered human remains as food debris in several rock shelters. At Qaranipuqa, human bone is a consistent component of the deposits from Layer O, which suggests that cannibalism was an early cultural trait in Fiji from 2500 BP that cooccurred with a population movement inland and the occupation of easily defendable hilltops (Best 1984:562). It is apparent, though, that most fortified sites were utilised post-1500 BP, and became common after 600 BP. Measured archaeologically, both conflict and cannibalism became increasingly prevalent in the past 500 years. Some support for this is in the skeletal record of conflict that shows it is absent-to-rare in human remains from Sigatoka dating to the first millennium AD (Pietrusewsky et al. 1994), but cannibalism is frequent in human remains associated with fortified sites on Waya Island that date to the past 300 years (Rechtman 1992).

\section{References}

Anderson A.J. and Clark, G.R. 1999. The age of Lapita settlement in Fiji. Archaeology in Oceania 34: 31-39.

Anderson, A., Clark, G. and Worthy, T. 2000. An inland Lapita site in Fiji. Journal of the Polynesian Society 109(3): 311-316.

Balouet, J.C. and Olson, S.L. 1987. An extinct species of giant pigeon (Columbidae: Ducula) from archaeological deposits on Wallis (Uvea) Island, South Pacific. Proceedings of the Biological Society of Washington 100(4): 769-775.

Baumel, J.J. and Witmer, L.M. 1993. Osteologia. In: Baumel, J.J., King, A.S., Breazile, J.E., Evans, H.E. and Vanden Berge, J.C. (eds), Handbook of avian anatomy: Nomina Anatomica Avium, Second Edition. Publications of the Nuttall Ornithological Club 23: 45-132. Cambridge, Massachusetts.

Best, S. 1981. Excavations at Site VL 21/5 Naigani Island, Fiji, a preliminary report. Department of Anthropology, University of Auckland.

Best, S. 1984. Lakeba: The prehistory of a Fijian island. Unpublished PhD thesis, University of Auckland, Auckland.

Birks, L. 1973. Archaeological excavations at Sigatoka dune site, Fiji. Bulletin of the Fiji Museum No.1.

Birks, L. and Birks, H. 1978. Archaeological excavations at site VL 16/81, Yanuca Island, Fiji. Oceanic Prehistory Records No. 6. University of Auckland.

Burley, D.V. 1998. Tongan archaeology and the Tongan past, 2850-150 B.P. World Archaeology 12(3): 337-392.

Burley, D.V. 2005. Mid-sequence archaeology at the Sigatoka Sand Dunes with interpretive implications for Fijian and Oceanic culture history. Asian Perspectives 44: 320-348.

Burley, D.V. 2007. In search of Lapita and Polynesian Plainware settlements in Vava'u, Kingdom of Tonga. In: Bedford, S., Sand, C. and Connaughton, S.P. (eds), Oceanic explorations: Lapita and Western Pacific settlement, pp. 187-198. Terra Australis 26. ANU EPress, Australian National University. 
Clark, G.R. and Anderson, A.J. 2001. The pattern of Lapita settlement in Fiji. Archaeology in Oceania 36: 77-88.

Clark, G.R., Anderson, A.J. and Matararaba, S. 2001. The Lapita site at Votua, northern Lau Islands, Fiji. Archaeology in Oceania 36: 134-145.

Clark, G. 2000. Mid-sequence isolation in the Fijian archipelago 2500-1000 BP. Bulletin of the IndoPacific Prehistory Association 19(3): 152-158.

Cochrane, E.E. 2006. Archaeology in the Yasawa Islands, Western Fiji: A report on fieldwork from 1978 to 2003. Domodomo 18(1-2): 1-67.

Cochrane, E.E., Pietrusewsky, M. and Douglas, M.T. 2004. Culturally modified human remains recovered from an earth oven internment on Waya Island, Fiji. Archaeology in Oceania 39: 54-59.

Davidson, J., Hinds, E., Holdaway, S. and Leach, F. 1990. The Lapita site of Natunuku, Fiji. New Zealand Journal of Archaeology 12: 121-155.

Davidson, J.M. and Leach. B.F. 2001. The strandlooper concept and economic naivete. In: Clark, G.R., Anderson, A.J. and Vunidilo, T. (eds), The archaeology of Lapita dispersal in Oceania, pp.115-123. Terra Australis 17, Pandanus Press, Canberra.

DeGusta, D. 1999. Fijian cannibalism: Osteological evidence from Navatu. American Journal of Physical Anthropology 110: 215-241.

DeGusta, D. 2000. Fijian cannibalism and mortuary ritual: Bioarchaeological evidence from Vunda. International Journal of Osteoarchaeology 10: 76-92.

Field, J.S. 2004. Environmental and climatic considerations: A hypothesis for conflict and the emergence of social complexity in Fijian prehistory. Journal of Anthropological Archaeology 23: 79-99.

Gifford, E.W. 1951. Archaeological excavations in Fiji. University of California Anthropological Records 13: 189-288.

Grant-Mackie, J.A., Bauer, A.M. and Tyler, M.J. 2003. Stratigraphy and herpetofauna of Me Aure cave (site WMD007), Mondou, New Caledonia. In: Sand, C. (ed), Pacific archaeology: Assessments and prospects, pp. 295-306. Les Cahiers de l' Archéologie en Nouvelle-Calédonie Number 15, New Caledonia.

Green, R.C. 1996. Prehistoric transfers of portable items during the Lapita horizon in remote Oceania: A review. Bulletin of the Indo-Pacific Prehistory Association 15: 119-130.

Groube, L.M. 1971. Tonga, Lapita pottery, and Polynesian origins. Journal of the Polynesian Society 80(3): 278-316.

Jones, D.N., Dekker, R.W.R.J. and Roselaar, C.S. 1995. The megapodes, Megapodiidae. Bird families of the world. Oxford University Press, Oxford.

Jones, S., Steadman, D.W. and O’Day, P.M. 2007. Archaeological investigations on the small islands of Aiwa Levu and Aiwa Lailai, Lau Group Fiji. Journal of Island and Coastal Archaeology 2: 72-98.

Keogh, J.S., Edwards, D.L., Fisher, R.N., and Harlow, P.S. 2008. Molecular and morphological analysis of the critically endangered Fijian iguanas reveals cryptic diversity and a complex biogeographic history. Philosophical Transactions of the Royal Society of London B 363: 3413-3426.

Hunt, T.L. 1980. Toward Fiji's past; archaeological research on southwestern Viti Levu. Unpublished MA thesis, University of Auckland.

Nunn, P.D. 2007. Echoes from a distance: Research into the Lapita occupation of the Rove Peninsula, southwest Viti Levu, Fiji. In: Bedford, S., Sand, C. and Connaughton, S.P. (eds), Oceanic explorations: Lapita and Western Pacific settlement, pp. 163-176. Terra Australis 26. ANU EPress, Australian National University.

Nunn, P.D., Ishimura, T., Dickinson, W.D., Katayama, K., Thomas, F., Kumar, R., Matararaba, S., Davidson, J. and Wothy, T. 2007. The Lapita occupation at Naitabale, Motiriki Island, Central Fiji. Asian Perspectives 46: 96-132.

Palmer, B. 1965. Excavations at Karobo, Viti Levu. New Zealand Archaeological Association Newsletter 8: 26-33. 
Pietrusewsky, M., Douglas, M.T. and Ikehara-Quebral, R.M. 1994. The human osteology of the Sigatoka dune burials (Site VL 16/1) Viti-Levu, Fiji Islands. Unpublished report, University of Hawaii.

Pratt, H.D., Bruner, P.I., and Berrett, D.G. 1987. A field guide to the birds of Hawaii and the tropical Pacific. Princeton University Press, Princeton, New Jersey.

Pregill, G.K. and Worthy, T.H. 2003. A new iguanid lizard (Squamata, Iguanidae) from the late Quaternary of Fiji, Southwest Pacific. Herpetologica 59: 57-67.

Rechtman, R.B. 1992. The evolution of socio-political complexity in the Fiji Islands. Unpublished PhD thesis, Ann Arbor, Michigan.

Steadman, D.W. 1985. Fossil birds from Mangaia, southern Cook Islands. Bulletin of the British Ornithologists Club 105(2): 58-65.

Steadman, D.W. 1987. Two new species of rails (Aves: Rallidae) from Mangaia, Southern Cook Islands. Pacific Science 40: 27-43.

Steadman, D.W. 1989. New species and records of birds (Aves: Megapodiidae, Columbidae) from an archaeological site on Lifuka, Tonga. Proceedings of the Biological Society of Washington 102(3): 537-552.

Steadman, D.W. 1992. New species of Gallicolumba and Macropygia (Aves: Columbidae). Los Angeles County Museum of Natural History, Science Series 36: 329-348.

Steadman, D.W. 1993. Biogeography of Tongan birds before and after human impact. Proceedings of the National Academy of Sciences USA 90: 818-822.

Steadman, D.W. 1994. Bird bones from the To'aga site, Ofu, American Samoa: Prehistoric loss of seabirds and megapodes. University of California Archaeological Research Facility, Contributions 51: 217-228.

Steadman, D.W. 1995. Prehistoric extinctions of Pacific island birds: Biodiversity meets zooarchaeology. Science 267: 1123-1131.

Steadman, D.W. 1997. The historic biogeography and community ecology of Polynesian pigeons and doves. Journal of Biogeography 24: 737-753.

Steadman, D.W. 1999. The biogeography and extinction of megapodes in Oceania. In: Dekker, R.W.R.J., Jones, D.N. and Benshemesh, J. (eds), Proceedings of the Third International Megapode symposium. Zoologische Verhandelingen Leiden 327: 7-21.

Steadman, D.W. 2006a. Extinction and biogeography of tropical Pacific birds. University of Chicago Press, Chicago and London.

Steadman, D.W. 2006b. A new species of extinct parrot (Psittacidae: Eclectus) from Tonga and Vanuatu, South Pacific. Pacific Science 60: 137-145.

Steadman, D.W. 2006c. An extinct species of tooth-billed pigeon (Didunculus) from the Kingdom of Tonga, and the concept of endemism in insular landbirds. Journal of Zoology 268: 233-241.

Steadman, D.W. and Franklin, J. 2000. A preliminary survey of landbirds on Lakeba, Lau Group, Fiji. Ети 100: 227-235.

Steadman, D.W. and Kirch, P.V. 1990. Prehistoric extinction of birds on Mangaia, Cook Islands, Polynesia. Proceedings of the National Academy of Sciences USA 87: 9605-9609.

Steadman, D.W., Worthy, T.H., Anderson, A.J. and Water R. 2000. New species and records of birds from prehistoric sites on Niue, Southwest Pacific. Wilson Bulletin 112: 165-186.

Steadman D.W., G.K. Pregill and Burley, D.V. 2002. Rapid prehistoric extinction of iguanas and birds in Polynesia. Proceedings of the National Academy Sciences USA 99: 3673-3677.

Taylor, J.M., Calaby, J.H. and van Deusen, H.M. 1982. A revision of the genus Rattus (Rodentia: Muridae) in the New Guinea region. Bulletin of the American Museum of Natural History 173: 177-336.

Thomas, F.R., Nunn, P.D., Osborne, T., Kumar, R., Areki, F., Matararaba, S., Steadman, D. and Hope G. 2004. Recent archaeological findings at Qaranilaca Cave, Vanuabalavu Island, Fiji. Archaeology in Oceania 39: 42-49. 
Watling, D. 1982. Birds of Fiji, Tonga and Samoa, Millwood Press, Wellington, New Zealand.

White, P.J., Clark, G. and Bedford, S. 2000. Distribution, present and past, of Rattus praetor in the Pacific and its implications. Pacific Science 54(2): 105-117.

Worthy, T.H. 2000. The fossil megapodes (Aves: Megapodiidae) of Fiji with descriptions of a new genus and two new species. Journal of the Royal Society of New Zealand 30: 337-364.

Worthy, T.H. 2001a. A new species of Platymantis (Anura: Ranidae) from Quaternary deposits on Viti Levu, Fiji. Palaeontology 44: 665-680.

Worthy, T.H. 2001b. A giant flightless pigeon gen. et sp. nov. and a new species of Ducula (Aves: Columbidae), from Quaternary deposits in Fiji. Journal of the Royal Society of New Zealand 31: 763-794.

Worthy, T.H. and Anderson, A.J. 1999. Research on the caves of Viti Levu, Fiji, June 1997 - October 1998, and their significance for palaeontology and archaeology. Report to the Fiji Museum, ANH, RSPAS, ANU, Canberra, ACT 0200, Australia.

Worthy, T.H., Anderson, A.J. and Molnar, R.E. 1999. Megafaunal expression in a land without mammals - the first fossil faunas from terrestrial deposits in Fiji (Vertebrata: Amphibia, Reptilia, Aves). Senckenbergiana biologica 79(2): 237-242.

Worthy, T.H., Walter, R. and Anderson, A.J. 1998. Fossil and archaeological avifauna of Niue. Notornis 45: 177-190.

Wragg, G.M. and Weisler, M.I. 1994. Extinctions and new records of birds from Henderson Island, Pitcairn Group, South Pacific Ocean. Notornis 41: 61-70.

Wragg, G.M. 1995. The fossil birds of Henderson Island, Pitcairn Group: Natural turnover and human impact, a synopsis. Biological Journal of the Linnean Society 56: 405-414. 